\title{
A numerical study of steady viscous flow past a circular cylinder
}

\author{
By BENGT FORNBERG
}

Department of Applied Mathematics, California Institute of Technology, Pasadena, California 91125, U.S.A.

(Received 13 June 1979)

\begin{abstract}
Numerical solutions have been obtained for steady viscous flow past a circular e- ]inder at Reynolds numbers up to 300. A new technique is proposed for the boundary condition at large distances and an iteration scheme has been developed, based on Newton's method, which circumvents the numerical difficulties previously encountered around and beyond a Reynolds number of 100. Some new trends are observed in the solution shortly before a Reynolds number of 300 . As vorticity starts to recirculate back from the end of the wake region, this region becomes wider and shorter. Other flow quantities like position of separation point, drag, pressure and vorticity distributions on the body surface appear to be quite unaffected by this reversal of trends.
\end{abstract}

\section{Introduction}

The problem of viscous incompressible flow past a circular cylinder has for a long time received much attention, both theoretically and numerically. In spite of many numerical methods and calculations, the Reynolds number $R e=100$ (based on the diameter) appears to be the upper limit for which complete, steady flow fields have been reliably determined. There are many reasons for the continuing interest in this problem and in attempts to carry numerical calculations to still higher Reynolds numbers. One of these reasons is that it is a good model problem for flows past other bodies of practical importance. Steady solutions for the circular cylinder become experimentally unstable around $R e=40$. Use of flow control methods to stabilize unstable solutions could lead to important new classes of flows, which at first may be studied more easily numerically. Many difficulties are encountered in attempts to analytically describe the complete flow field. We believe that numerical methods can provide further information on the limiting properties of the steady flow for increasing Reynolds numbers. Questions such as the asymptotic development of the recirculation region (wake bubble), drag, position of separation point, vorticity and pressure distributions, etc., are all open and they are relevant to the understanding of high-Reynoldsnumber flows.

Brodetsky (1923) suggests a solution for infinite Reynolds number in which vortex sheets bound an infinite wake region containing stagnant flow. This solution is often referred to as the Helmholtz-Kirchhoff free streamline model because of their introduction of vortex sheets (Helmholtz 1868; Kirchhoff 1869). Both an infinite wake and a finite drag is in agreement with experimentally and numerically observed trends for low Reynolds numbers (up to 100 to 200). Batchelor (1956) gives however arguments against these features of the limit and suggests that the vorticity inside the wake in the 
limit need not be zero but can take a constant value on each side of the line of symmetry. Solutions of this kind (if they exist) would allow for a finite wake and therefore no drag on the body.

The most notable phenomenon we observe at high Reynolds numbers $(R e>260)$ is a shortening of the wake region with vorticity being convected into its interior. Our upper limit, $R e=300$, is not high enough however to establish whether or not this new trend will persist.

In a recent theoretical work, Smith (1979) assumes that the wake length will increase to infinity and that the flow will tend to the Brodetsky limit with no vorticity inside the wake. There are of course profound differences between the consequences he draws from these assumptions and our results for $R e>260$. However, there are also differences for much lower Reynolds numbers as for example his figure 3 for the skin friction shows. Our numerical results agree well with the comparisons he quotes and confirm therefore the discrepency illustrated in that figure.

The flow problem is formulated mathematically in $\S 2$. In $\S 3$ we discuss the most frequently encountered numerical difficulties. Section 4 discusses boundary conditions at large distances. The final numerical method is presented in $\$ 5$ with the obtained results discussed in $\$ 6$. This numerical method was employed for Reynolds numbers 20-300. Some results for Reynolds numbers 2-10 are also presented, although they were obtained by a different technique, using a direct iteration scheme based on fast Poisson solvers. Since many methods work successfully in that range and the flow patterns are well known, this low-Reynolds-number method will not be discussed further.

The extensive numerical calculations for high Reynolds numbers were performed on the Control Data Corporation STAR 100 computer, located at the CDC Service Center in Arden Hills, Minnesota. We wish to express our gratitude to Control Data Corporation for making this computer system available to us. The solution of large banded linear systems of equations was the most time-consuming part of the present calculations. These solutions ran about 200 times faster on the CDC STAR 100 than on the Caltech IBM 370/158 computer. The IBM machine was used for Reynolds numbers from 2 up to 10, for preliminary tests of the high-Reynolds-number method on very small grids and for the final data processing and graphical presentation.

\section{Mathematical formulation}

In terms of stream function $\Psi^{\prime}$ and vorticity $\omega$, satisfying

$$
\begin{gathered}
u=\partial \Psi / \partial y, \quad v=-\partial \Psi / \partial x, \\
\omega=\partial v / \partial x-\partial u / \partial y,
\end{gathered}
$$

the Navier-Stokes equations can be formulated

$$
\begin{gathered}
\Delta \Psi+\omega=0, \\
\Delta \omega+\frac{R e}{2}\left\{\frac{\partial \Psi}{\partial x} \frac{\partial \omega}{\partial y}-\frac{\partial \Psi}{\partial y} \frac{\partial \omega}{\partial x}\right\}=0,
\end{gathered}
$$

where $\Delta=\partial^{2} / \partial x^{2}+\partial^{2} / \partial y^{2}$ and the Reynolds number $R e$, based on the diameter $d$, is 

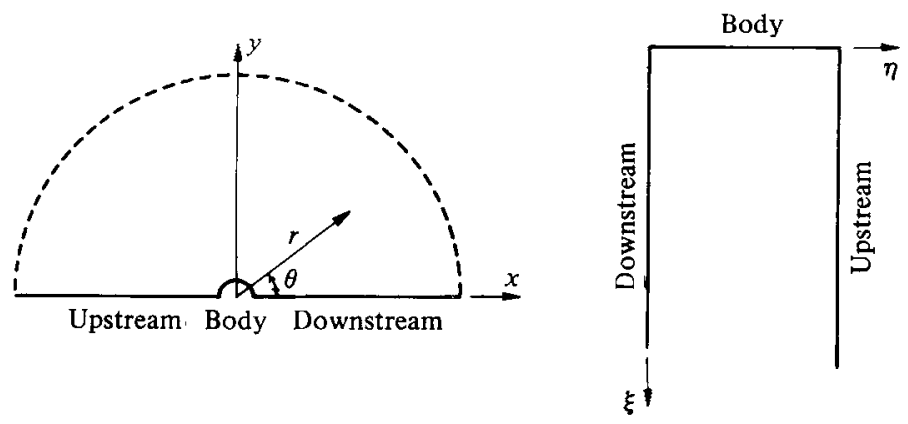

Figure 1. The $x, y$ and $\xi, \eta$ plane.

$R e=U d / \nu$. The quantity $U$ is the free-stream velocity and $\nu$ is the kinematic coefficient of viscosity. We will from now on assume that $U=\frac{1}{2} d=1$.

It is convenient to work both numerically and theoretically with the deviation from uniform flow

instead of with $\Psi$.

$$
\psi(x, y)=\Psi(x, y)-y
$$

A polar co-ordinate system can be introduced by the conformal transformation

$$
\xi+i \eta=\frac{1}{\pi} \ln (x+i y) .
$$

(The variables $\xi$ and $\eta$ are connected to the usual polar co-ordinates $r$ and $\theta$ by $r=e^{\pi \xi}$ and $\theta=\pi \eta$.) The Navier-Stokes equations take in these co-ordinates the form

$$
\begin{gathered}
\Delta \psi=-\pi^{2} r^{2} \omega \\
\Delta \omega=\frac{R e}{2}\left\{\pi r\left(\cos \theta \frac{\partial \omega}{\partial \xi}-\sin \theta \frac{\partial \omega}{\partial \eta}\right)-\frac{\partial \psi}{\partial \xi} \frac{\partial \omega}{\partial \eta}+\frac{\partial \psi}{\partial \eta} \frac{\partial \omega}{\partial \xi}\right\}
\end{gathered}
$$

here

$$
\Delta=\partial^{2} / \partial \xi^{2}+\partial^{2} / \partial \eta^{2}
$$

We assume symmetry and consider only the upper half-plane. The half-plane minus the cylinder gets mapped into the semi-infinite strip $0 \leqslant \eta \leqslant 1, \xi \geqslant 0$ (figure 1).

On the surface of the body, the boundary conditions are

$$
\begin{aligned}
& \psi(0, \eta)=-\sin (\pi \eta), \\
& \frac{\partial \psi(0, \eta)}{\partial \xi}=-\pi \sin (\pi \eta),
\end{aligned}
$$

corresponding to vanishing normal and tangential derivatives of $\Psi$. Symmetry gives $\psi=0$ and $\omega=0$ as boundary conditions on $\eta=0$ and $\eta=1$. Numerically, two boundary conditions must also be supplied at some outer limit $r_{\infty}$. The choice of these outer conditions will be discussed in detail later. One commonly used simple choice is to use the 'free stream', i.e. $\psi=0, \omega=0$ on this boundary. As we will see, this choice is very unsatisfactory. Even when applied hundreds of radii away from the body, it will lead to significant errors in the flow field (in particular in the vorticity) right up to the body surface. 


\section{Numerical difficulties for increasing Reynolds numbers}

Sooner or later numerical attempts to simulate accurately the flow at increasingly high Reynolds numbers run into factors which limit further progress. The existence and the nature of such factors have rarely been discussed in the numerical literature on this problem. We believe however that the following list contains the main problem in this respect.

1. How to implement boundary conditions at large distances.

2. How to implement boundary conditions at the surface of the body.

3 . How to get a reliable rate of convergence for the numerical iterations (a rate which does not deteriorate seriously with increasing Reynolds numbers).

4. How to approximate the vorticity transport equation in a way which (i) is stable, (ii) is at least accurate to second order, ideally allowing Richardson extrapolation (or deferred correction) to fourth-order accuracy, (iii) makes the overall iteration scheme convergent.

5. How to get adequate but not wasteful resolution of all the different scales.

The item 1 above will be the subject of a detailed discussion in $\S 4$. Here, we will only make brief comments on the other items and then wait until $\S 5$, where we will see how the proposed method handles them.

Problem 2 arises because we have two boundary conditions for $\psi$ and none for $\omega$, where one for each variable might have proved easier to work with. Many different techniques have been tried at the boundary. It is our impression that convergence problems have been present at this boundary in many cases.

We believe that the limiting factor in most previous work is contained in problem 3. The physical problem becomes unstable with respect to unsymmetric disturbances around $R e=40$. With symmetry imposed, stability persists much longer (for how long is not known). Numerical methods for the steady symmetric problem have in most cases involved iterations between the stream function and vorticity equations. Such iterations introduce an artificial time in which instabilities can be encountered quite early without the symmetric problem being unstable in real time. We believe this artificial time instability is the main reason why accurate calculations have not yet reached high Reynolds numbers. Our approach to this problem is to solve the coupled stream function and vorticity equations rather than using one equation at a time in some iterative manner.

The vorticity equation in steady calculations has often been solved as an elliptic system with a method based on the successive over-relaxation method. Local diagonal dominance must then be assured for the difference approximation of the vorticity transport process. This has led to such methods as upwind differencing (see Roache 1976, p. 64 for references). In its simplest form, using simple uncentred approximations for $\partial \omega / \partial \xi$ and $\partial \omega / \partial \eta$, the local accuracy is only first order, which computationally is extremely inefficient. Different techniques to maintain second-order accuracy have been given. Allen \& Southwell (1955) give a scheme which can be described as a continuous transition between upwind differencing and centred approximations. The diagonal dominance is barely maintained at all instances. In the limit of step sizes going to zero, the approximations become centred, and the whole method is therefore formally of second order. Dennis (1973) has studied this method for finite step sizes and has found that it works surprisingly well and may even allow 
Richardson extrapolation. Another idea to obtain second-order accuracy is to extend the approximation width in the upstream direction. Leonard (1979) has described such a method. The general experience seems to be however that upwind techniques compare unfavourably with centred approximations with respect to accuracy. There is no generally accepted simple scheme which satisfies all the requirements listed under problem 4.

The flow field shows a mixture of different scales for high Reynolds numbers. There is close to the body a thin boundary layer, which separates and extends downstream. Far away from the body, a narrow wake contains a sharp perturbation from free stream while, in all directions far out, a slow but non-trivial perturbation exists. Usual polar co-ordinate systems which are dense enough to resolve the wake far out will be very wasteful in other directions. Our choice of grids will exploit the fact that $\omega \ll 1$ and $\psi$ is very smooth and governed by a simple linear equation in most of the outer field.

It is not always easy to tell which of these difficulties has been the most pronounced in previous steady calculations. We have indicated some guesses in table 1, which summarizes a number of previous contributions to the problem.

\section{Far-field boundary conditions}

Two boundary conditions must be supplied at some finite, large distance $r_{\infty}$ from the cylinder (or at infinity if a transformation has been made which brings infinity to a finite distance). Since both the stream function and the vorticity equations are of elliptic nature, it appears natural to supply one condition for each of the variables along each edge.

Asymptotic formulas are known for $\psi$ and $\omega$ as the distance $r$ increases to infinity (see for example Imai 1951). The leading terms are

$$
\begin{aligned}
& \psi=\frac{C_{D}}{2}(\eta-\operatorname{erf} Q) \\
& \omega=-\frac{C_{D} \operatorname{Re}}{4 \sqrt{\pi}} \frac{Q}{r} e^{-Q^{2}},
\end{aligned}
$$

with

$$
Q=\left(\frac{1}{2} \operatorname{Rer}\right)^{\frac{1}{2}} \sin \frac{1}{2} \pi \eta, \quad \operatorname{erf} Q=2 \pi^{-\frac{1}{2}} \int_{0}^{Q} e^{-s^{2}} d s
$$

$C_{D}$ is the drag coefficient. In $x, y$ co-ordinates, this means that, to leading order and at large distances, the difference $\psi$ between stream function $\Psi$ and free stream $y$ looks like a simple source with equal outflow in all directions balanced by an inflow in a narrow region behind the body. At high Reynolds numbers, this simple picture is valid first at very large distances. Figure 2 show plots of $\psi$ for $R e=2$ and $R e=200$. The ultimate directions of some of the lines (given by (11) when $C_{D}$ has first been calculated) have also been marked. As the Reynolds number increases, $C_{D}$ decreases and therefore, according to (11), the strength of the radial outflow. The flow behind the body is almost stagnant and the inflow in the $\psi$ variable does not decrease correspondingly. This causes the large circulation in $\psi$ that we see in figure $2(b)$. 


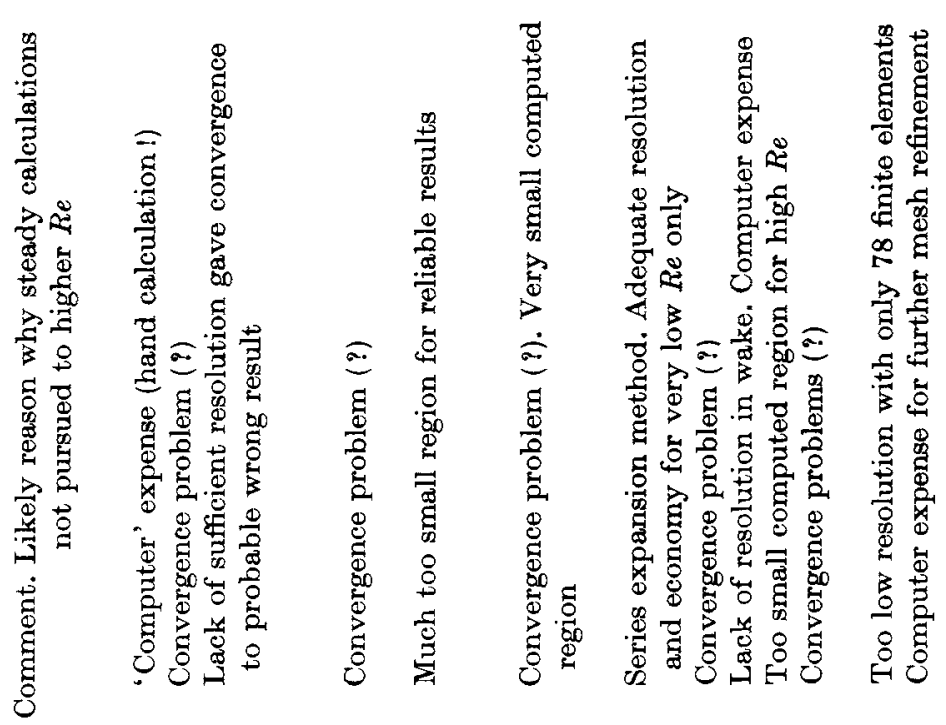

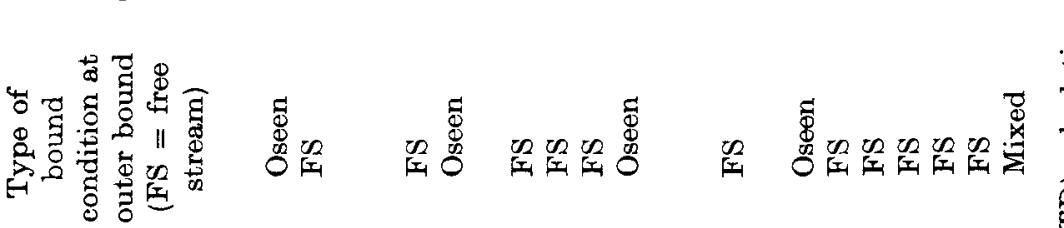
章 曼 $\circ$

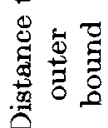

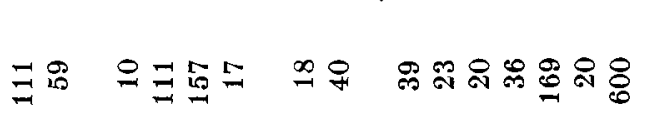
矛

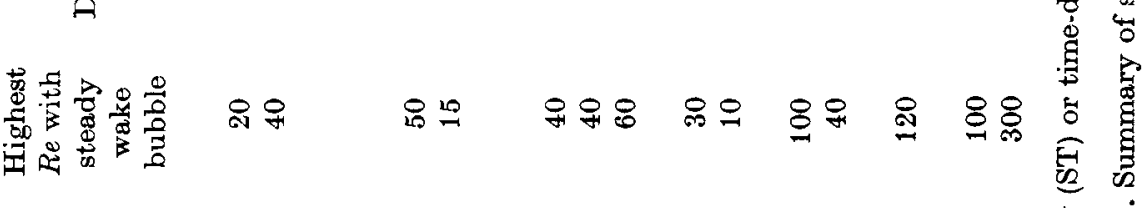

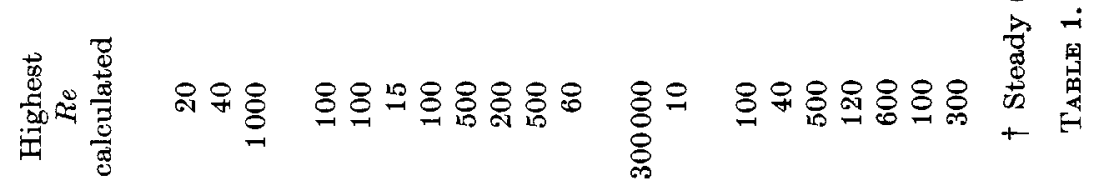
范

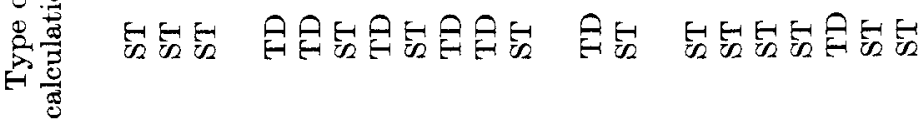

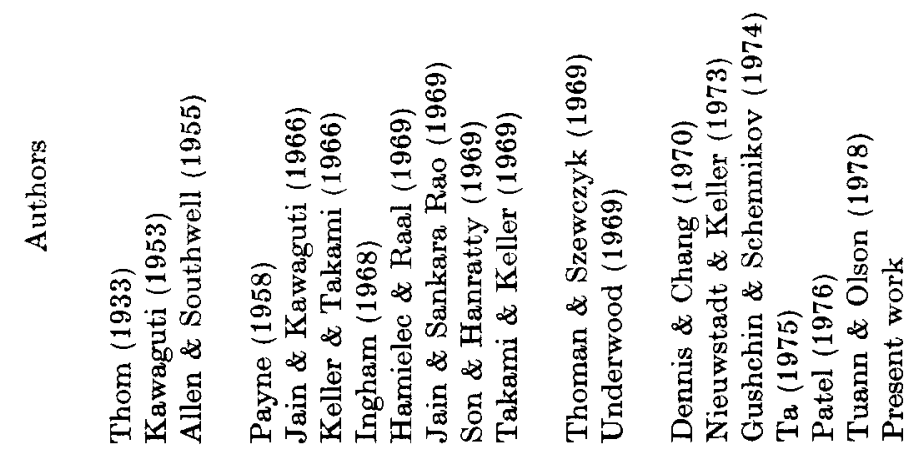




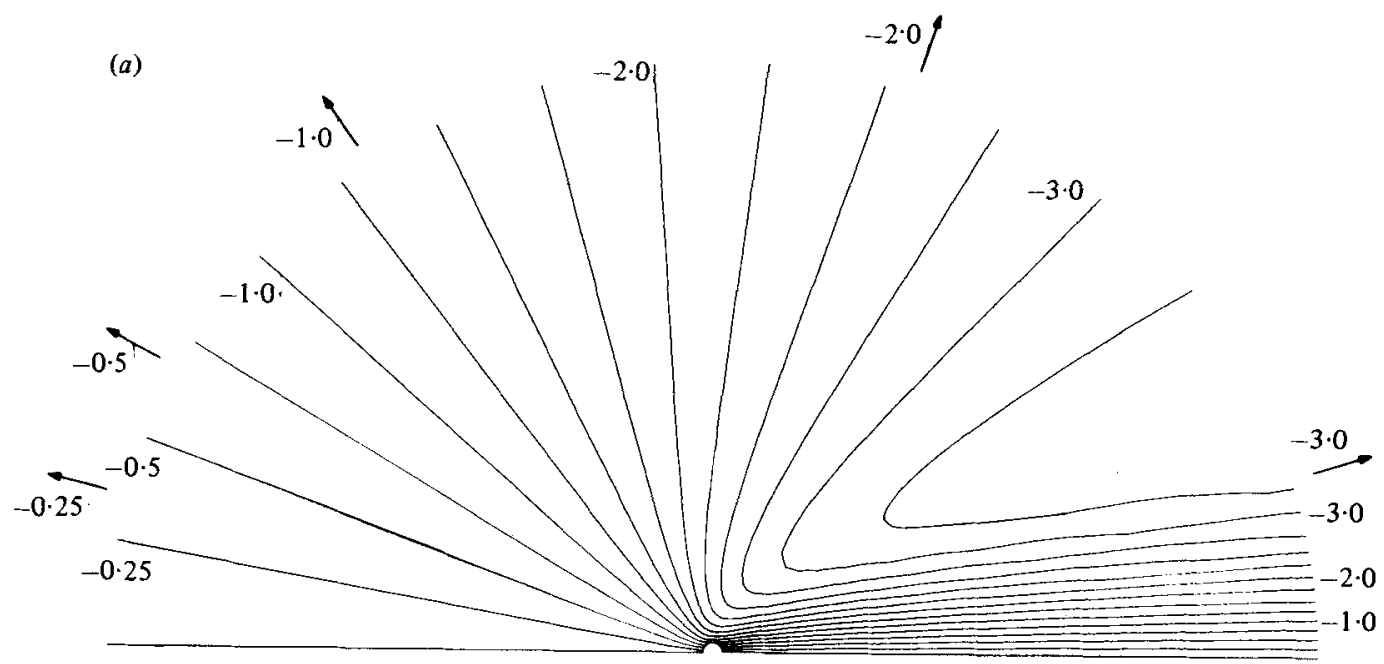

(b)
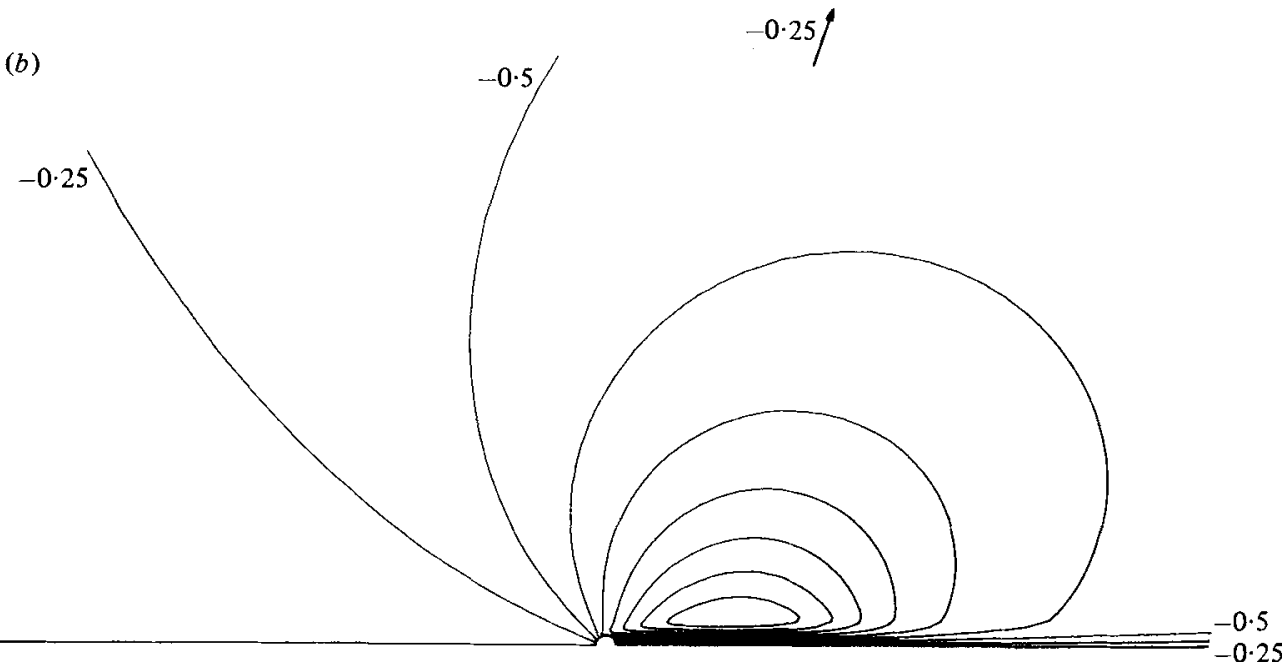

Figure 2. The $\psi$ field for $(a) R e=2$ and $(b) R e=200$.

The vorticity far from the body is concentrated in a thin streak downstream with maximum strength inversely proportional to the distance. Since the question of boundary condition for $\omega$ turns out to be much simpler than the condition for $\psi$, we consider that case first.

\section{Boundary condition for $\omega$ at $r_{\infty}$}

Vorticity is transported along streamlines and dissipated by viscosity. It is only because of the dissipative term that a boundary condition at the outflow side is needed. If wrong boundary values are supplied, the introduced errors will decrease exponentially upwind from this boundary. The only concern we need have is purely numerical. The computed values must not fall apart into staggered mesh oscillations far into the region. That would normally happen if values for $\omega$ are given on the boundaries and all 
approximations are centred and accurate to second order. Upwind approximations of the vorticity transport would solve the problem, but with a loss of accuracy. Our choice is to use centred approximations, but impose $\partial \omega / \partial \xi=0$ on the boundary (by equating the values on the last two mesh lines). As we noted, the problem did not need any information from the outer boundary and this method prevents the possibility of staggered mesh oscillations.

\section{Boundary condition for $\psi$ at $r_{\infty}$}

The question of boundary conditions for $\psi$ is more involved. The quantity $\psi$ satisfies in $\xi, \eta$ co-ordinates

$$
\Delta \psi+\pi^{2} r^{2} \omega=0
$$

Actual physical information has to be supplied through the outer boundary, and it will propagate immediately to the interior. A distance $r=100$ corresponds to $\xi=1.47$ and $r=10^{6}$ to $\xi=4 \cdot 40$. The use of a large $r_{\infty}$ together with inaccurate boundary values does not give a reasonable accuracy. Also, as we will see, we want to avoid numerical calculations for large values of $r$ because of increasing differences between the scales in the problem.

We will consider four different choices of boundary condition for $\psi$.

(a) Free stream (using $\psi=0$ on the outer edge).

(b) One term of the Oseen approximation (equation (11)).

(c) Normal derivative zero $(\partial \psi / \partial \xi=0)$.

(d) 'Mixed condition' connecting $\psi$ and $\partial \psi / \partial \xi$ on the boundary.

We will briefly discuss the justification for each of these choices and then use two tests to assess their accuracy and usefulness. These tests are:

(i) to plot $\psi$ and $\omega$ when we move $r_{\infty}$ in and out and see how much the flow picture changes;

(ii) to display the maximum vorticity on the body surface as a function of $r_{\infty}$ with the different choices of boundary condition.

\section{(a) Free stream}

We know that $\psi$ for large $\xi$ will tend to a linear function in $\eta$ (with a jump at $\eta=0$ ) and that the values of $\psi$ satisfy a Poisson equation in $\xi$ and $\eta$. The use of the free stream, i.e. imposing $\psi=0$ at some small $\xi_{\infty}$, seems very crude. Nevertheless, as table 1 showed, this has been used in most cases even for very small values of $r_{\infty}$. Therefore the method has to be studied for accuracy. Figure $3(a)$ shows the result of test 1 at Reynolds number $R e=2$ using $r_{\infty}=23 \cdot 1$ and $r_{\infty}=91 \cdot 5$. We can easily conclude that large errors have been introduced. In particular, the vorticity field has changed even up to the body surface. Figure $4(a)$ shows that the maximum vorticity on the body surface is as much as $20 \%$ in error with respect to $r_{\infty}=23 \cdot 1$. Figures $4(b, c)$ show that large errors also persist for high Reynolds numbers. (Note that larger $r_{\infty}$ have been used in figures $4 b, c$.)

\section{(b) One term of the Oseen approximation}

The use of this boundary condition requires knowledge of the drag coefficient $C_{D}$. This can be evaluated from the current approximation of the flow field at each iteration by means of a line integral around the body. Figures $2(a, b)$ indicate that this boundary 

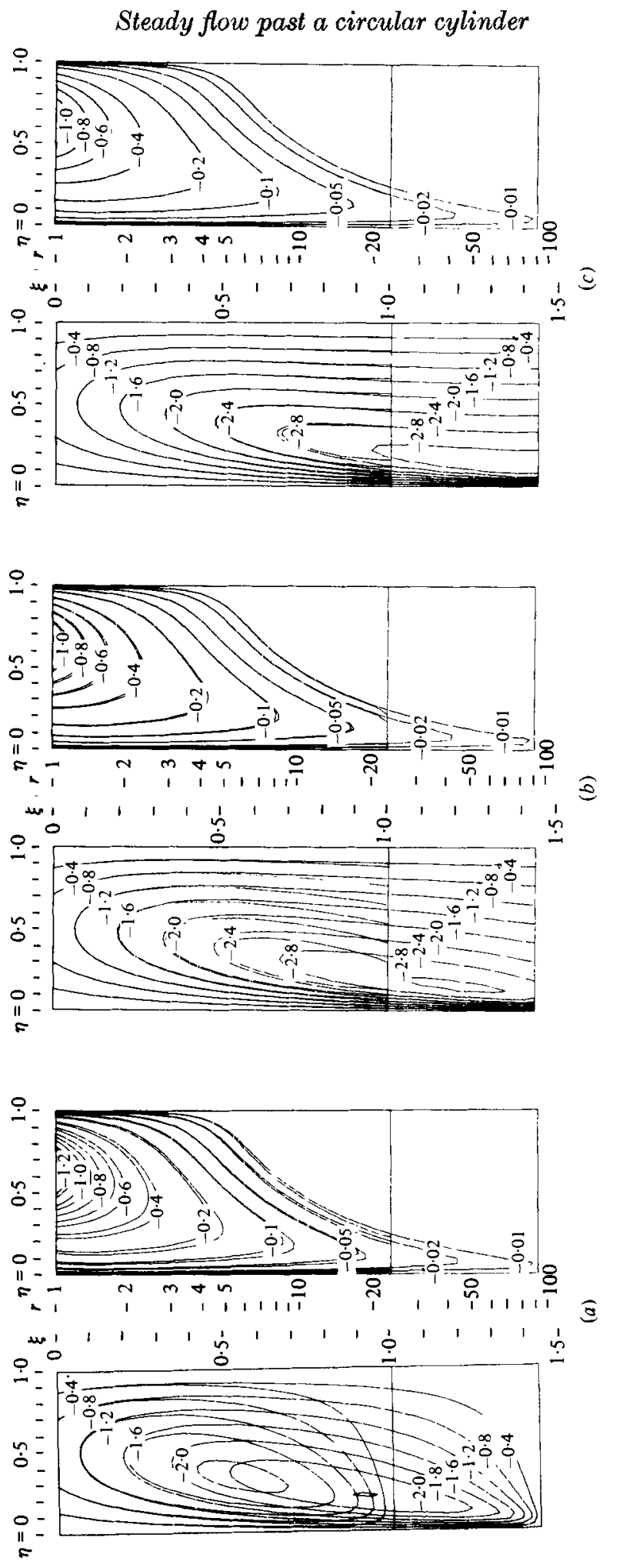

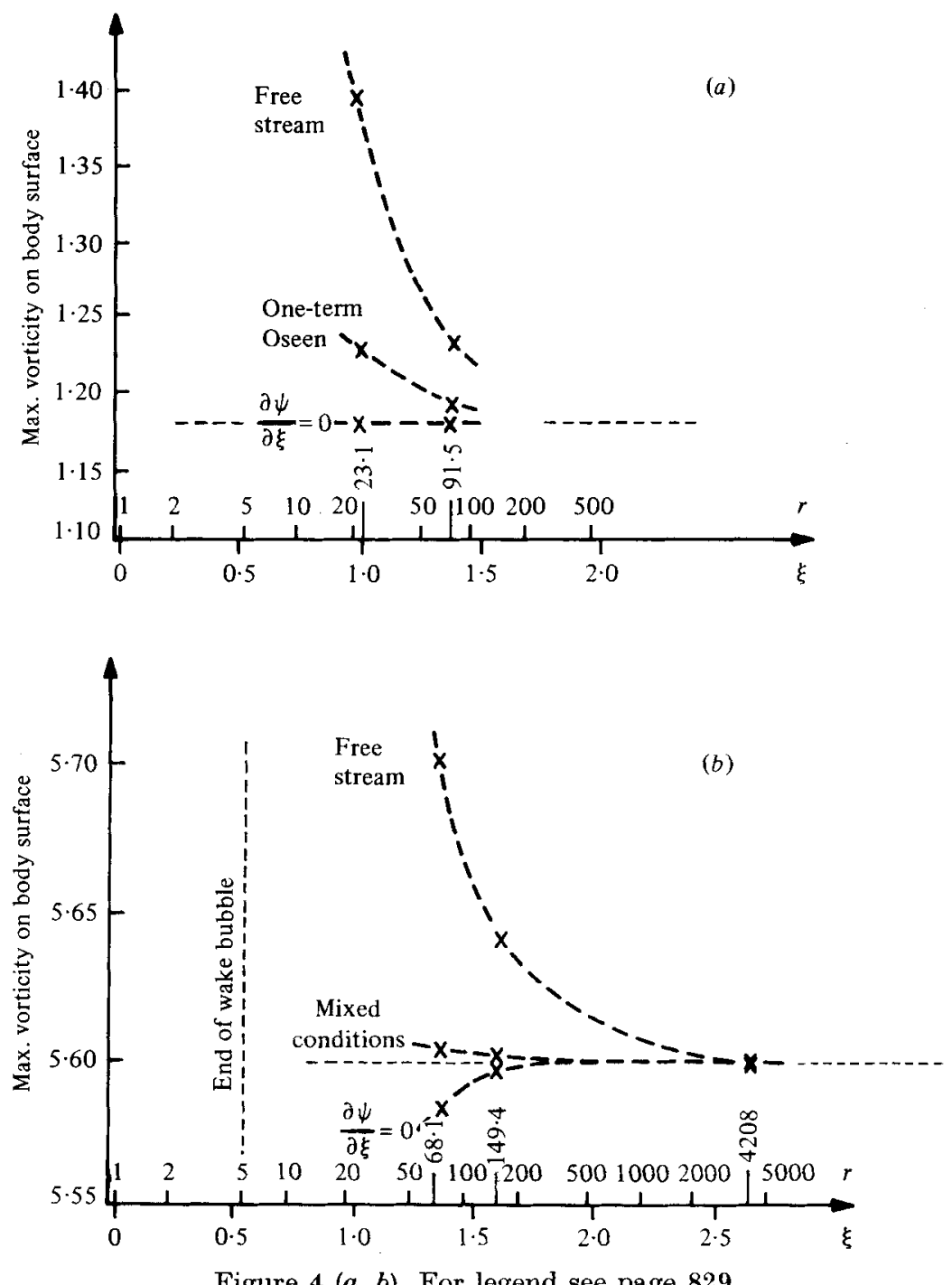

Figure $4(a, b)$. For legend see page 829 .

condition can be expected to be accurate at most for very low Reynolds numbers. Figure $3(b)$ and $4(a)$ show however quite significant errors already for $R e=2$. We did not try this method for higher Reynolds numbers.

\section{(c) Normal derivative zero}

This condition is as easy to apply as free stream (for instance, it requires no evaluation of $C_{D}$ ). It is a 'softer' condition than $\psi=0$ and is consistent with the picture of equilines of $\psi$ eventually going in the $\xi$ direction (radially out from the body everywhere apart from in the wake where it is radially in towards it). Test number 1 in figure $3(c)$ shows this method to be superior to the previous methods at a low Reynolds number. Both the justification and the actual performance of this boundary condition decrease however with increasing Reynolds numbers as figures 2 and $4(a, b)$ show. 


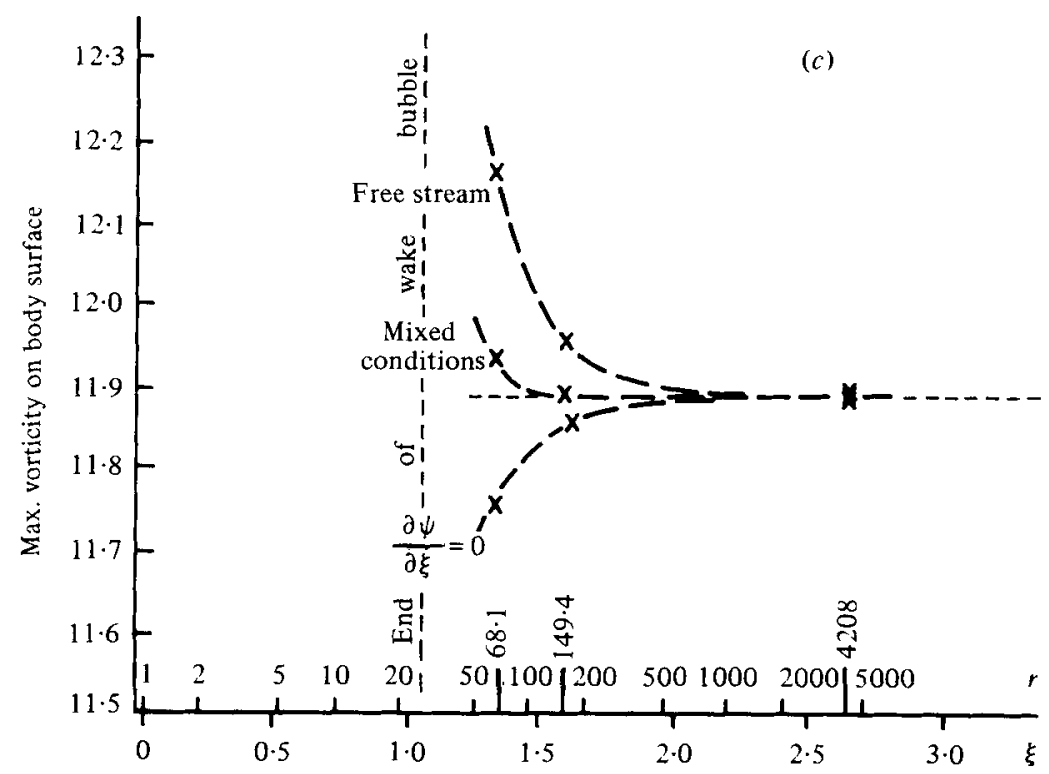

Figure 4. Comparison between different outer boundary conditions for $\psi$ at $(a) R e=2$, (b) $R e=40$ and (c) $R e=200$.

We feel that, for practical purposes, this condition completely solves the problem of an easy and accurate boundary condition for $\psi$ at Reynolds numbers up to about 40 .

\section{(d) 'Mixed condition' method}

Let us start the description of this technique with some observations. In $\xi, \eta$ co-ordinates, the vorticity decays like $O\left(e^{-\pi \xi}\right)$ along the $\xi$ axis. This vorticity enters as righthand side in the equation (7) for $\psi$. These small values of $\omega$ are scaled up by the factor $r^{2}$ which is large at large distances. We know that the net effect of this is that $\psi$ converges to a linear function of $\eta$ (with a jump at $\eta=0$ ) as $r$ tends to infinity. From figure $2(b)$ however we know that, for reasonable distances and high Reynolds numbers, $\psi$ behaves in a non-trivial way for all values of $\eta$, not just for $\eta \approx 0$. It is desirable to apply a boundary condition so close to the body that the thin transition around $\eta=0$ and the slow variation for all $\eta$ can both be resolved by the same grid. The further out the boundary condition is applied, the more severe will the mixing of scales become.

Figure 5 shows what $\omega$ and $\psi$ look like in $\xi, \eta$ co-ordinates at $R e=200$. A dotted line is drawn where we would like to implement the boundary condition. According to our previous discussion of boundary conditions for $\omega$, we can expect to have fairly accurate values of $\omega$ available close to this line. There are two reasons why $\psi$, determined from the linear equation (7), takes non-zero values below this line. Let us write $\psi=\psi_{1}+\psi_{2}$ with the two components $\psi_{1}$ and $\psi_{2}$ corresponding to the two reasons below.

(i) There is a thin streak of vorticity along the $\xi$ axis. It decays and gets thinner but the factor $r^{2}$ in (7) enhances the influence of vorticity at large distances. The net effect of this streak of vorticity is that $\psi$ for large values of $\xi$ converges to a function of $\eta$ with a jump of $\eta=0$ (as given by equation (11)). In the thin region where vorticity is 


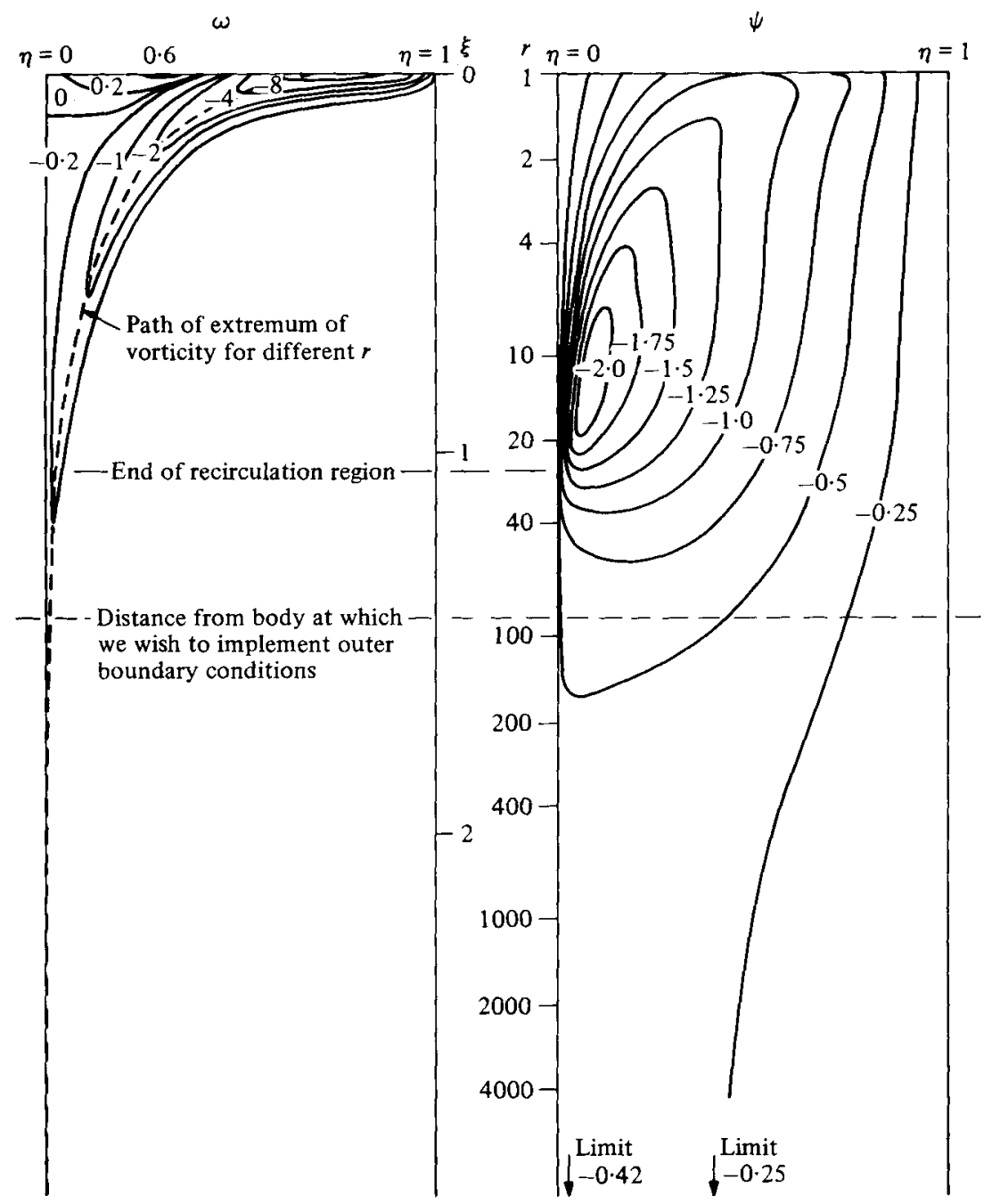

Frgure 5. Contours of $\omega$ and $\psi$ at $R e=200$.

present, $\partial^{2} \psi_{1} / \partial \xi^{2}$ is very small compared to $\partial^{2} \psi_{1} / \partial \eta^{2}$ so $\psi_{1}$ satisfies approximately

for constant $\xi$.

$$
\frac{\partial^{2} \psi_{1}}{\partial \eta^{2}}=-\pi^{2} r^{2} \omega
$$

(ii) The values of $\psi$ close to the body influence the values of $\psi$ far out since they are coupled by equation (7). Far out, we can think of this influence as a potential flow with $\psi_{2}$ satisfying

$$
\frac{\partial^{2} \psi_{2}}{\partial \xi^{2}}+\frac{\partial^{2} \psi_{2}}{\partial \eta^{2}}=0
$$

(The right-hand side in ( 7 ) is already taken care of in equation (13) for $\psi_{1}$.) The boundary conditions for $\psi_{2}$ are $\psi_{2}=0$ at the three sides $\eta=0, \eta=1, \xi=\infty$ and unknown at $\xi=\xi_{\infty}$. As figures $2(b)$ and 5 clearly show, this term $\psi_{2}$ is important along the full 
extent of the outer boundary. The Oseen approximation assumes the flow in the wake to depend on the drag only and fails to describe this part $\psi_{2}$ of the solution for $\psi$.

These observations lead us to the following method:

(a) We suppose a guess $\psi^{*}$ for the variable $\psi$ is available at $\xi=\xi_{\infty}$.

(b) With $\omega$ known at two mesh lines close to $\xi_{\infty}$, we can use (13) to solve for $\psi_{1}$ (two-point boundary value problems) on these lines and thus get an approximation for $\partial \psi_{1} / \partial \xi$ at $\xi=\xi_{\infty}$.

(c) We evaluate $\psi_{2}=\psi^{*}-\psi_{1}$ at $\xi=\xi_{\infty}$. Since $\psi_{2}$ satisfies (14), we can easily (Fourier decomposition) find $\partial \psi_{2} / \partial \xi$ at $\xi=\xi_{\infty}$. (Note that this does not require any numerics to be performed beyond $\xi_{\infty}$.)

(d) Given the guess $\psi^{*}=\psi_{1}+\psi_{2}$ we have thus found $\partial \psi_{1} / \partial \xi$ and $\partial \psi_{2} / \partial \xi$, i.e. given $\psi$ we know $\partial \psi / \partial \xi$. This is exactly the right form of a mixed condition to be imposed on (7) at $\xi=\xi_{\infty}$. We can put this relation in the form: Given $\psi$ on the next to the last mesh line, we can find it on the last line. That formulation is ideally suited for iterative methods to solve (7).

Figures $4(b, c)$ show how this condition works for $R e=40,200$ respectively. We did not try it for Reynolds numbers lower than 40 since the simpler condition $\partial \psi / \partial \xi=0$ worked sufficiently well there.

As a summary, it appears that the use of the simple $\partial \psi / \partial \xi=0$ for Reynolds numbers up to about 40 and the mixed condition for higher Reynolds numbers solves the question of boundary condition for $\psi$ at large distances.

Dennis (1976) also points out the insufficiency of the free stream as the outer boundary condition and proposes a technique based on matching $\omega, \partial \omega / \partial \xi, \psi$ and $\partial \psi / \partial \xi$ between an inner region governed by the Navier-Stokes equations and an outer region governed by a simplified version of the equations. The approach suggested for the outer region differs in many respects from our observations of the flow pattern and our conclusions from it. In particular computations are performed throughout this outer region (to find both $\psi$ and $\omega$ ), and the simplified equations have terms in $\partial^{2} / \partial \xi^{2}$ dropped. The term $\partial^{2} \psi / \partial \xi^{2}$ is assumed to be small compared to $\partial^{2} \psi / \partial \eta^{2}$ at large distances. This leads to parabolic equations which can be marched for both $\psi$ and $\omega$.

Both the economy and the justification of such an approach seem unclear to us in the light of our previous discussion.

\section{Numerical method for steady solutions}

The main problem that a method for steady solutions at high Reynolds numbers must solve is the one of assured fast rate of convergence for the discrete approximating equations. Since the real physical problem may be unstable, any iteration technique which can resemble artificial time may have convergence problems. This danger is entirely circumvented with the use of Newton's method for the discrete nonlinear system of equations. With an initial guess close enough to a simple solution, quadratic convergence is assured. Many iterative methods fail to converge as moving wave patterns appear in the artificial time. Because of the quadratic convergence, such waves cannot appear in this method. The price to be paid for this guaranteed performance is a comparatively high computational cost. This is no longer a major concern with the introduction of extremely powerful array processors.

We have already remarked that the problem involves different scales. Vorticity is 


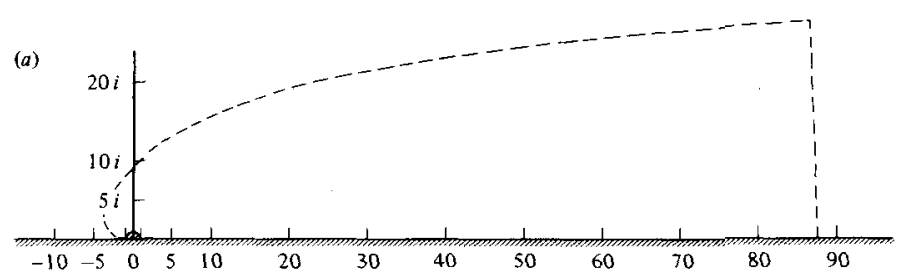

(b)
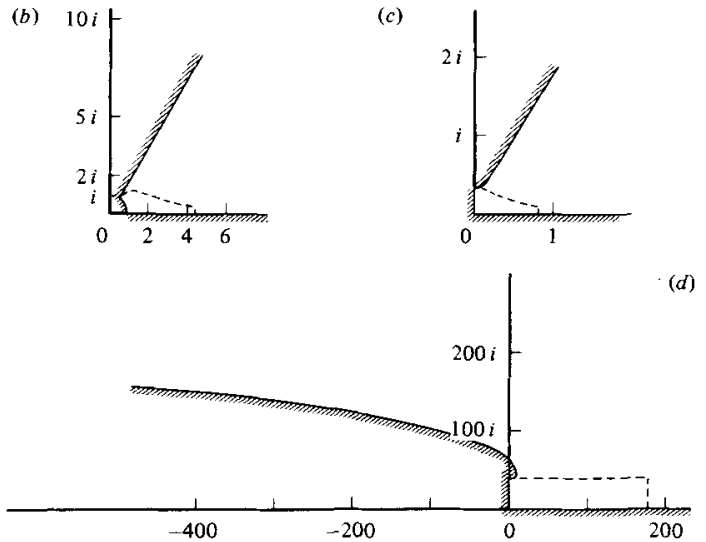

(c)

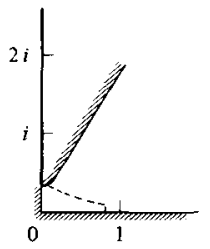

FiquRE 6. Steps in the mapping of the inner region, $c=0 \cdot 2 .(a) x$ plane;

(b) $\rho$ plane; (c) $z$ plane; (d) $\zeta$ plane.

only present at the body and in a streak downstream from it. This region is the only one where the problem is truly nonlinear. Outside that region, we have only to solvo

$$
\Delta \psi=0
$$

The solution to this equation only changes on a large scale. This clearly suggests the use of two different grids with different numerical methods on them.

The dotted region in figure $6(a)$ is a suitable computational region for the nonlinear part of the flow field. A conformal mapping of a region of such shape to a rectangle can be achieved explicitly. For instance, setting $\rho=x^{\frac{1}{3}}$ maps the upper half-plane minus the unit circle (figure $6 a$ ) into the region in figure $6(b)$, and $z=c(\rho-1 / \rho)$ maps the segment of the unit circle onto the imaginary axis and leads to region in the figure $6(c)$. In these figures and in the following computations, the constant $c$ was given the value $0 \cdot 2$. Finally, we want to obtain a mapping which resembles the original $x, y$ system far downstream and the $z$ co-ordinates close to the surface. This suggests a final step $\zeta=z\left(1+z^{2}\right) c^{-3}$. The real and imaginary axis are kept from the $z$ plane but the cubic term cancels the cube root in the initial step (figure $6 d$ ).

We put a rectangular grid in the $\zeta$ plane as is shown dotted in figure $6(d)$. Its images in the other planes are shown in figures $6(a-c)$. (Changing the constant $c$ affects the width of the mapped wake region in the $x$ plane.) The complete mapping from $x$ to $\zeta$ can be written as

$$
\left.\begin{array}{l}
z=c\left(x^{\frac{1}{3}}-1 / x^{\frac{1}{3}}\right), \\
\zeta=\frac{1}{c^{3}} z\left(1+z^{2}\right),
\end{array}\right\}
$$


and it can be inverted in closed form (choosing principal branches) by

$$
\left.\begin{array}{l}
t=c^{3 \zeta}, \\
\alpha=\left\{\left(\frac{t^{2}}{4}+\frac{1}{27}\right)^{\frac{1}{2}}+\frac{t}{2}\right\}^{\frac{1}{3}}, \\
\beta=\alpha-\frac{1}{3 \alpha}, \\
x=\left\{\frac{\beta}{2 c}+\left(\left(\frac{\beta}{2 c}\right)^{2}+1\right)^{\frac{1}{2}}\right\}^{3} .
\end{array}\right\}
$$

With the notation $x=\xi+i \eta$ and $\zeta=\alpha+i \beta$ equations (7) and (8) take, in the $\zeta$ plane, the forms

$$
\begin{gathered}
\frac{\partial^{2} \psi}{\partial a^{2}}+\frac{\partial^{2} \psi}{\partial \beta^{2}}+\omega / M=0 \\
\frac{\partial^{2} \omega}{\partial \alpha^{2}}+\frac{\partial^{2} \omega}{\partial \beta^{2}}+\frac{R e}{2}\left\{\frac{\partial \psi}{\partial \alpha} \cdot \frac{\partial \omega}{\partial \beta}-\frac{\partial \psi}{\partial \beta} \cdot \frac{\partial \omega}{\partial \alpha}-\left(\frac{\partial \omega}{\partial \alpha} \cdot \frac{\partial \alpha}{\partial \xi}+\frac{\partial \omega}{\partial \beta} \cdot \frac{\partial \beta}{\partial \xi}\right) / M\right\}=0
\end{gathered}
$$

where $M=(\partial \alpha / \partial \xi)^{2}+(\partial \beta / \partial \xi)^{2}$. The functions $\partial \alpha / \partial \xi$ and $\partial \beta / \partial \xi$ can be evaluated as the real and imaginary parts of the complex function

$$
\frac{\partial \zeta}{\partial x}=\frac{\partial \zeta}{\partial z} \cdot \frac{\partial z}{\partial \rho} \cdot \frac{\partial \rho}{\partial x}=\frac{\left(1+3 z^{2}\right)(\rho+1 / \rho)}{3 x c^{2}} .
$$

In a further transformation step, the grid was compressed close to the body and stretched far out by the change

$$
\alpha=c_{1}\left(e^{c_{z} \gamma}-1\right)
$$

where $c_{1}$ and $c_{2}$ are constants $(0.008$ and $0 \cdot 118$ respectively in our work). A rectangular region in the $\gamma, \beta$ plane is now discretized equidistantly. On this rectangular grid in the $\gamma, \beta$ plane, we approximate the transformed Navier-Stokes equations in the straightforward way with centred, second-order approximations. On this inner grid, $\psi$ was given on all the boundaries and also $\partial \psi / \partial \gamma$ on the body surface. For $\omega$, no condition was given on the surface, $\omega=0$ on top and bottom boundaries and $\partial \omega / \partial \gamma=0$ on the right side boundary (large distance). When we used our fine grid $(65 \times 114$ points in the $\beta$ and $\gamma$ directions respectively, corresponding to an outer boundary approximately 600 radii from the body), the condition involving the normal derivative $\partial \psi / \partial \gamma$ at the surface was approximated using a centred approximation with an artificial point inside the body. Use of (18) at the surface allowed this inside point to be eliminated, giving a relation between $\omega$ at the surface and $\psi$ at the mesh line nearest the surface. The less accurate method of approximating $\partial \psi / \partial \gamma$ by a one-sided stencil out from the body (and no artificial point inside the body) is also satisfactory in this problem, and was used in our calculations on the coarse inner grids $(33 \times 44,33 \times 52$ and $33 \times 86)$.

The discretizations we have described lead to a large coupled nonlinear system of equations with the same number of equations as unknowns. Newton's method is now used to solve this system. To describe the structure of the Jacobian matrix for this system, we need some additional notation. Assuming the grid in the $\gamma, \beta$ plane is of size $N \times M$, we let

$$
\omega_{1}, \omega_{2}, \omega_{3}, \ldots, \omega_{N}
$$




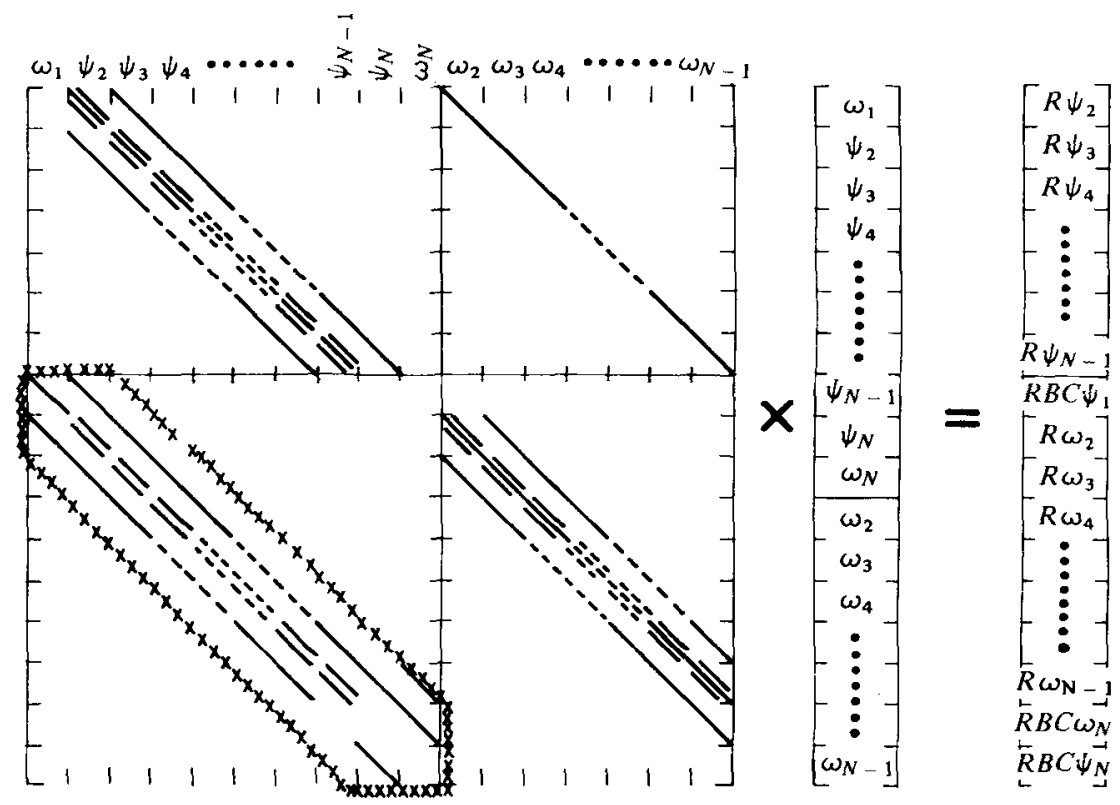

Figure 7. Structure of the linear system in Newton's method.

be vectors of length $M-2$ containing the values of $\omega$ along the $M-2$ interior points in the $\beta$ direction on the $N$ different grid lines. (For example $\omega_{1}$ contains the values of the vorticity on the body surface.) Similarly, we use

$$
\psi_{1}, \psi_{2}, \psi_{3}, \ldots, \psi_{N}
$$

for the function $\psi$. Further, let

$$
\begin{aligned}
& R \omega_{2}, R \omega_{3}, \ldots, R \omega_{N-1}, \\
& R \psi_{2}, R \psi_{3}, \ldots, R \psi_{N-1}
\end{aligned}
$$

be vectors containing the residuals in the vorticity and stream function equations centred along lines 2 to $N-1$. Finally,

$$
R B C \psi_{1}, R B C \omega_{N}, R B C \psi_{N}
$$

are the residual vectors for the boundary conditions on lines 1 and $N$. (Since $\psi_{1}$ is known, we do not enter $\psi_{1}$ as an unknown. $R B C \psi_{1}$ refers to the second condition, $\partial \psi / \partial \gamma$ given.)

The Jacobian coefficient matrix takes a shape which directly reflects the shapes of the approximating stencils. The particular ordering of the equations and the unknowns in the linear system shown in figure 7 may at first look irregular. This ordering, however, leads to a coefficient matrix which easily simplifies to a form well suited to numerical solution. As a first step in solving the system, we superpose multiples of the equations in the first $N-2$ (block) rows to eliminate all entries in the bottom right major block. The bottom left major block then assumes a 13-diagonal form, which is completely confined inside the dotted region in figure 7 . Figure 8 shows the structure of this band 


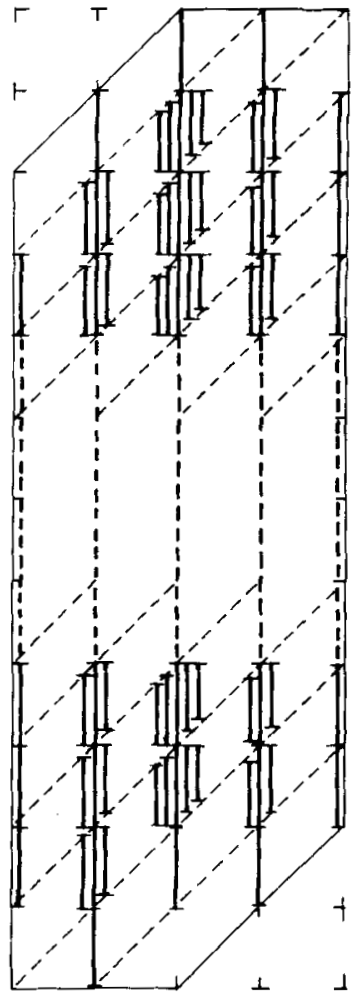

Figure 8. Structure of the 13-diagonal banded coefficient matrix.

\begin{tabular}{|c|c|c|c|c|}
\hline & & \multirow[b]{2}{*}{$\begin{array}{l}\text { General grid size } \\
\qquad M \times N\end{array}$} & \multicolumn{2}{|c|}{ Grids used in present work } \\
\hline & & & $\begin{array}{l}\text { Coarse grid } \\
33 \times 48\end{array}$ & $\begin{array}{r}\text { Fine grid } \\
65 \times 114\end{array}$ \\
\hline $\begin{array}{l}\text { Size of reduced } \\
\text { banded system to } \\
\text { solve }\end{array}$ & $\begin{array}{l}\text { Bandwidth } \\
\text { Number of } \\
\text { equations }\end{array}$ & $\begin{array}{l}4 \times M-7 \\
(M-2) \times(N+1)\end{array}$ & 125 & 253 \\
\hline $\begin{array}{l}\text { Computer time for } \\
\text { numerical solution }\end{array}$ & $\begin{array}{l}\text { CDC STAR-100: } \\
\text { LU-decomposition } \\
\text { Back substitution } \\
\text { IBM 370/158: } \\
\text { (estimate) } \\
\text { LU-decomposition }\end{array}$ & - & $\begin{array}{l}2.55 \mathrm{~s} \\
0.22 \mathrm{~s}\end{array}$ & $\begin{array}{r}29 \cdot 33 \mathrm{~s} \\
1.25 \mathrm{~s}\end{array}$ \\
\hline
\end{tabular}

TABLE 2. Sizes and computer times for banded linear systems.

matrix (with the lines displaced sideways so that the diagonals go vertically down in the picture). The approximate cost of solving this banded system is shown in table 2 . The LU-decomposition for the $65 \times 114$ grid requires approximately $470 \times 10^{6}$ additions and multiplications. Using FORTRAN with no special optimization, this required $29 \cdot 33$ seconds (including all non-arithmetic operations like loop control, partial pivoting 


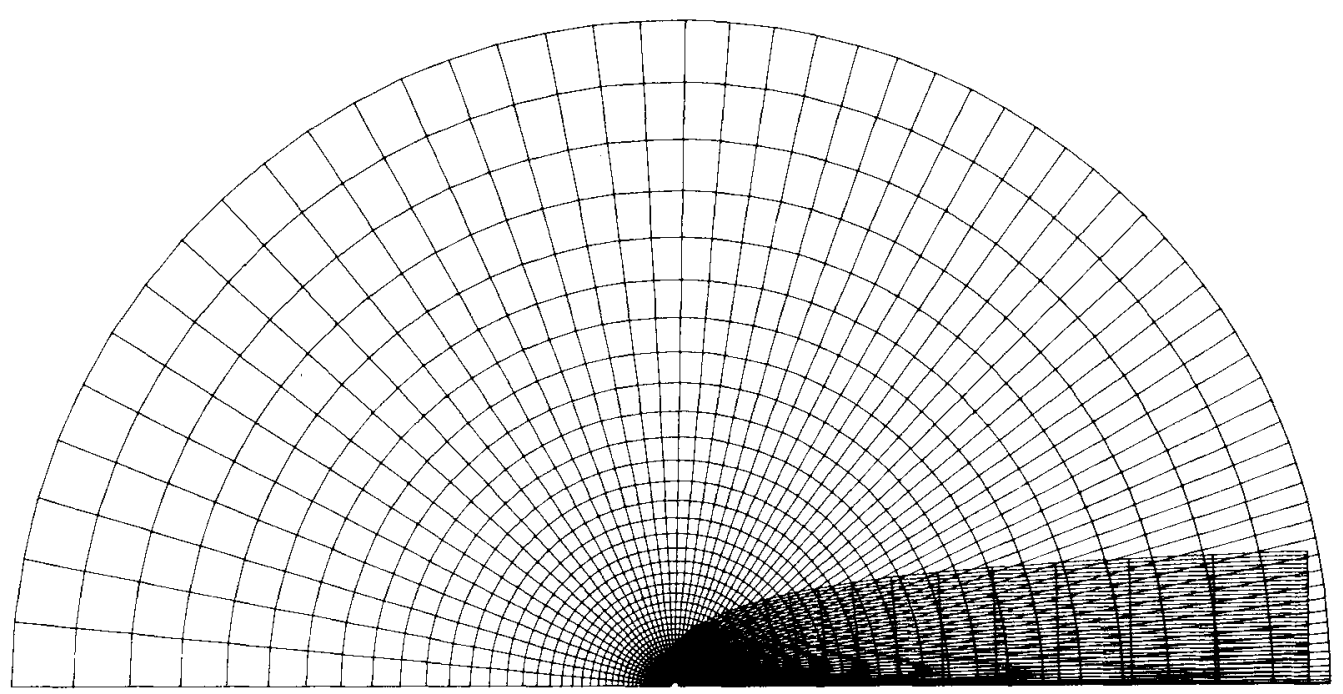

Figure 9. An example of the superposition of outer and inner grids.

etc.) on the CDC STAR 100, giving an average speed of about 16 Mflops (Million floating point operations per second, 64 bit wordlength).

The 13-diagonal system we obtained after the initial elimination could have been generated more directly first by analytically eliminating $\omega$ between (18) and (19) and then by approximating the resulting fourth-order biharmonic-type equation for $\psi$ with standard centred approximations. It is not clear which is the better method by which to generate the final banded system.

As we have observed earlier, $\psi$ behaves non-trivially in all directions from the body even at large distances. If $\omega$ is known inside the wake, $\psi$ can be found everywhere from the simple equation (7). To solve this equation, we use a modified polar grid $\xi, \eta^{\prime}$ with $\xi, \eta^{\prime}$ given by

$$
\xi+i \eta=\frac{1}{\pi} \ln (x+i y), \quad \eta^{\prime}=\eta^{\frac{1}{2}}
$$

On this grid, we discretize $\xi$ and $\eta^{\prime}$ equidistantly. Figure 9 shows a typical case of inner and outer grids superposed onto each other.

We can now describe the complete method. Assuming we have an initial guess for $\psi$ and $\omega$, we carry out the following steps:

1. Perform one Newton iteration on the inner grid. This gives an improvement in $\omega$ (and in $\psi$ ).

2. Interpolate $\omega$ to the outer grid using two-dimensional cubic splines (fourth-order accurate).

3. Solve the Poisson equation for $\psi$ on the outer grid (with the 'mixed' boundary condition at the outer boundary). This solution is obtained by 'black-red' ordered successive over-relaxation (SOR).

4. Interpolate these new $\psi$ values back to the edges of the inner grid. Return to step 1.

When the grid sizes become large, step 1 is the most expensive one. It also converges quadratically while the iterations between the grids only converge linearly. For these 
reasons, we perform only one Newton iteration for each outer iteration. In the step 4 , over- and under-relaxation can be used when the values of $\psi$ on the edge from the previous iteration are corrected with better approximations. The use of relaxation factors between 0.5 and 1.5 (rather than 1.0 corresponding to using only the new approximation) led in all cases to an overall convergence with a factor of about 10 during each full cycle over steps 1-4. Six to eight digits of accuracy in solving the nonlinear system (truncation errors are probably larger) thus required about eight iterations. Complete computer times, including all overhead, ranged from between 1 and 2 minutes for a $33 \times 48$ inner grid and between 6 and 8 minutes for a $65 \times 114$ inner grid. These times were independent of the Reynolds number.

Direct jumps in Reynolds numbers 20-40-100-130-160-200-230-260 were used with the $65 \times 114$ inner grid. In the proximity of Reynolds number 300 smaller continuation steps were needed: 260-275-285-290-295-297.5-300. If the initial guess was not sufficiently close to the solution, the Newton iterations diverged, first in the areas where the guess (the solution at a previous Reynolds number) was least accurate. This was in the region where vorticity recirculation was observed. The changes were there very rapid as a function of the Reynolds number. Since Reynolds number 300 also is close to the upper limit at which results are reliable due to truncation errors (calculations on a grid twice coarse began to deviate significantly shortly before Reynolds number 200) and since further mesh refinement would have been too costly, no effort was made to reach still higher Reynolds numbers. We found no indication of any kind that any principal problem was present, which might have prevented further increases in Reynolds number, had a finer grid been used.

We will now discuss how the proposed method handles the difficulties that were listed in $\S 3$.

1. Boundary conditions at large distances were discussed in detail in $\S 4$. We applied the 'mixed' boundary condition to the outer grid only. A Dirichlet condition, obtained by interpolation from the outer grid, was used for the inner grid. Since the outer grid was handled by SOR, the 'mixed' condition was easily implemented.

2. The problem with boundary conditions at the surface of the body was that we had two conditions for $\psi$ and none for $\omega$, when one for each variable might have been easier. With Newton's method, all that is required to obtain quadratic convergence is the correct number of equations, an isolated solution, and a sufficiently good initial guess. The fact that there was not one condition for each of the variables $\psi$ and $\omega$ has become completely irrelevant.

3. Quadratic convergence is assured in Newton's method for isolated solutions. This excludes every possibility that waves or similar features may move in artificial time during the iterations. (The linearly convergent outer-inner iteration to find boundary values for $\psi$ for the inner grid could conceivably have failed to converge.)

4. Since convergence on the inner grid has been assured, there is no longer any need to introduce special stencils or other methods to stabilize the vorticity transport approximation at the expense of the accuracy. Second-order centred approximations satisfy all the three requirements. Since the interpolation between the grids was fourth-order accurate, overall fourth-order accuracy could have been obtained by Richardson extrapolation.

5. The chosen grids, with the inner one refined at the body surface, seemed to give suitable resolution in all regions. It was possible to use a much cheaper numerical 
(a)

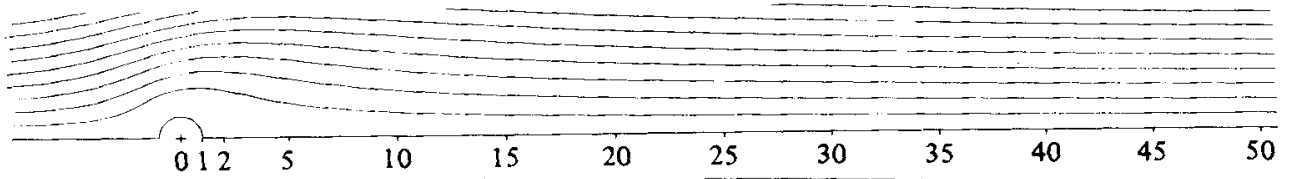

(b)

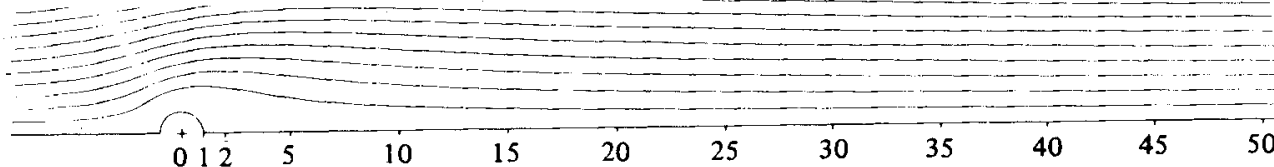

(c)

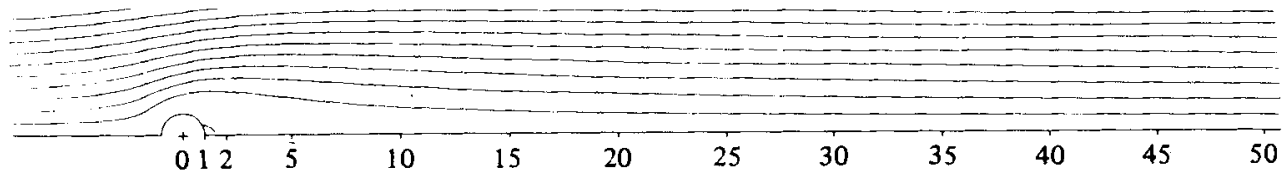

(d)

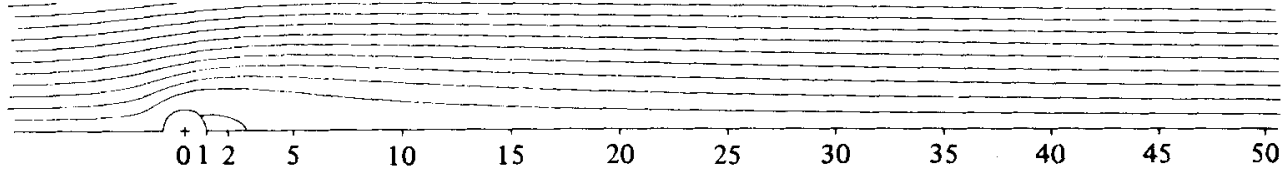

(e)

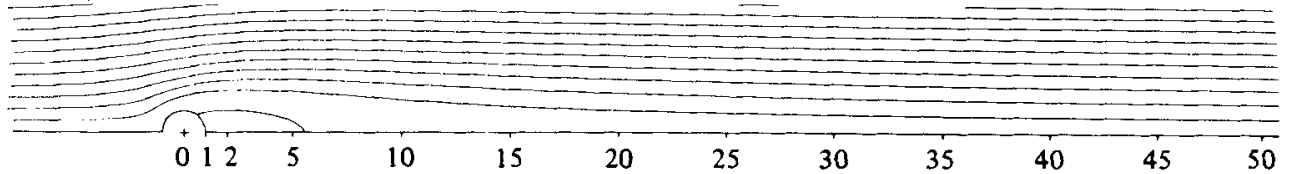

$(f)$

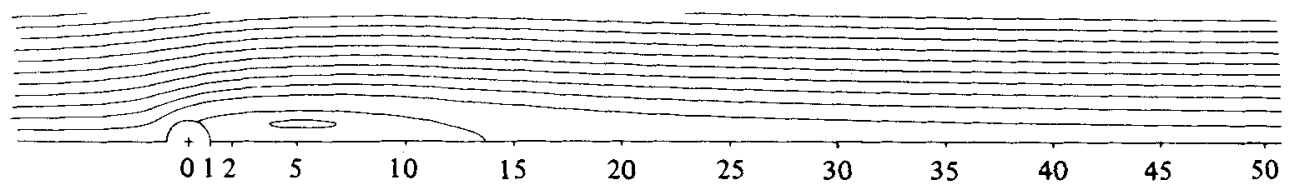

Figure $10(a-f)$. For legend see page 839.

technique in those regions where the equations were without special nonlinear difficulties.

\section{Results}

There are many questions on the limiting flow fields at high Reynolds numbers that remain open. Although this investigation does not solve any of them, distinct trends for increasing Reynolds numbers can be seen in several cases. The most striking feature we notice is a recirculation of vorticity from the end of the wake region, starting around a Reynolds number of 260 . This leads to quite sudden changes in some flow quantities like the length and the width of the wake region while other quantities, such as the vorticity and pressure distributions on the body surface and the drag, seem quite unaffected. The rest of this section contains a discussion of the results, mainly focusing on the numerical aspects rather than on the physical consequences of the observations. As was mentioned in the introduction, the results for $R e=2$ to 10 were not obtained by the present method, and are of comparatively low accuracy and graphical resolution.

Figures 10 and 11 show the streamlines and the vorticity fields for $R e=2$ to 300 . We notice a recirculation of vorticity, clearly developed beyond $R e=290$ but suggested 
$(g)$

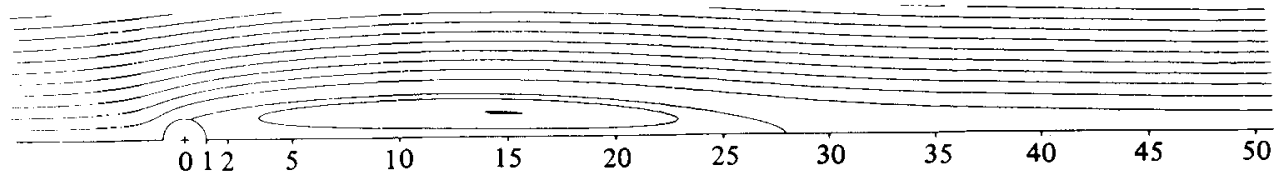

(h)

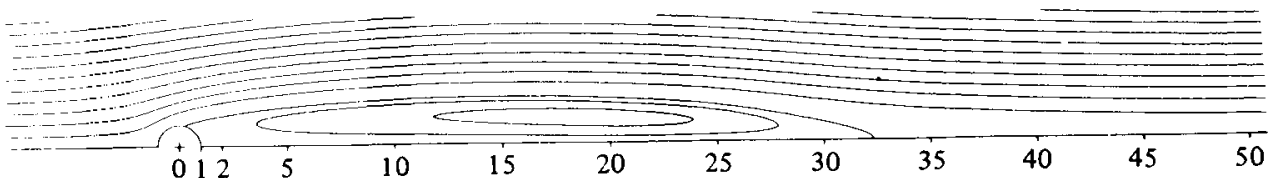

(i)

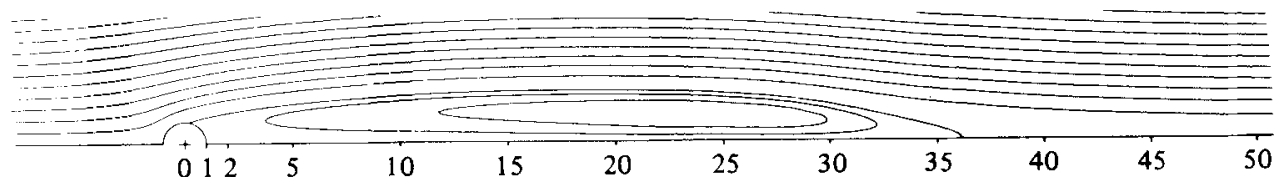

(j)

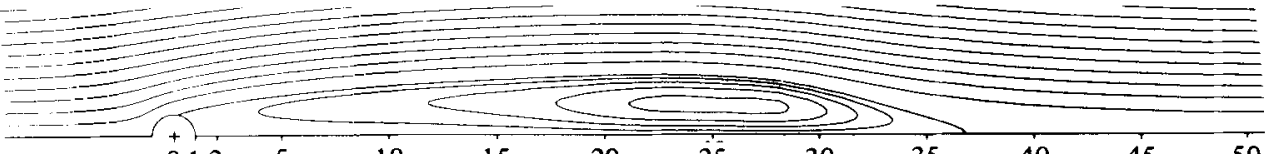

(k)

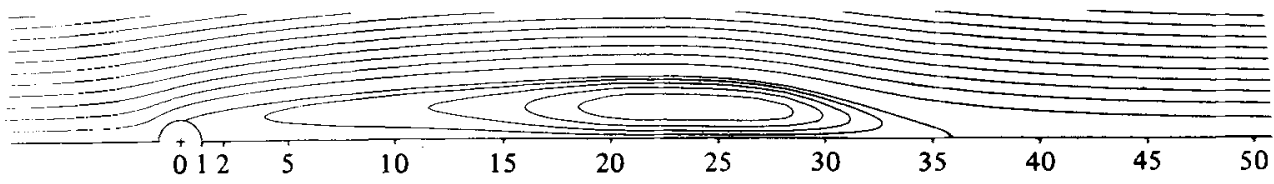

(l)

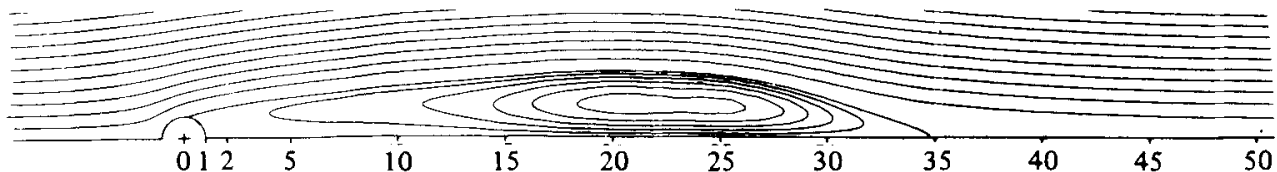

Figure 10. Streamlines at different Reynolds numbers. (a) $R e=2 ;$ (b) $R e=4$; (c) $R e=10$; (d) $R e=20 ;(e) R e=40 ;(f) R e=100 ;(g) R e=200 ;(h) R e=230 ;(i) R e=260 ;(j) R e=290$; (k) $R e=295$; (l) $R e=300$.

by the vorticity fields already at $R e=200,230$. This widening of the region with vorticity causes the separating streamline to close nearer the body. (The small 'island' of low vorticity in figure $11(l)$ should be connected with the thin streak approaching it from the left. The vertical mesh resolution was not sufficient for representing this streak continuously.)

To ensure as far as is possible that the observed features of the flow fields are real and not peculiarities of the discrete approximations, we checked the independence of these solutions to changes in the grid sizes. Halving the densities of both outer and inner grids from $129 \times 132,65 \times 114$, respectively, to $65 \times 52,33 \times 48$ (also, corresponding to a smaller total region) only offered sufficient resolution power up to a Reynolds number of around 150. Figures $12(a)-(h)$ compare the flow fields using these different grids for $R e=20$ and 200. The discrepancies are quite large at $R e=200$ but the figures show that the beginning of a trend towards a recirculation of vorticity, is present on both grids. At $R e=290$, only the fine grid could be used, but we have a parameter $c$ available in the conformal mapping for the inner grid. This constant $c$ was changed from 0.2 to 0.25 and 0.3 . This corresponds to halving the vertical extent of the inner grid (i.e. doubling the vertical grid density). Figures $13(a)-(f)$ show the effect 
(a)

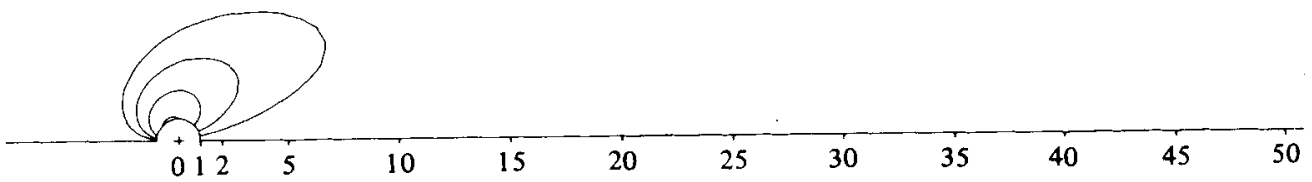

(b)

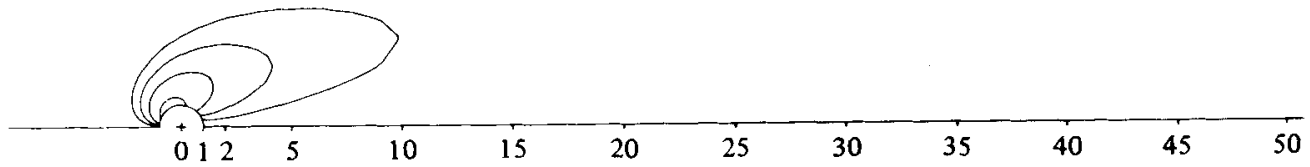

(c)

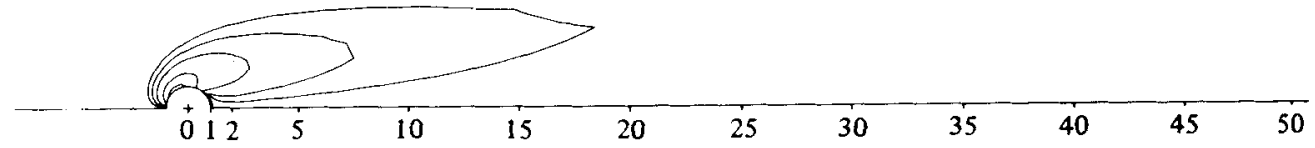

(d)

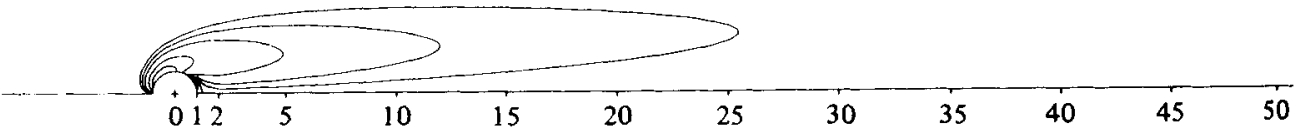

(e)

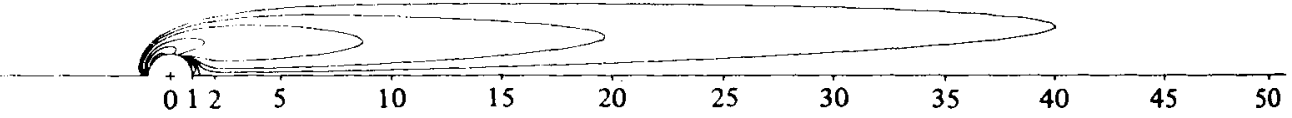

$(f)$

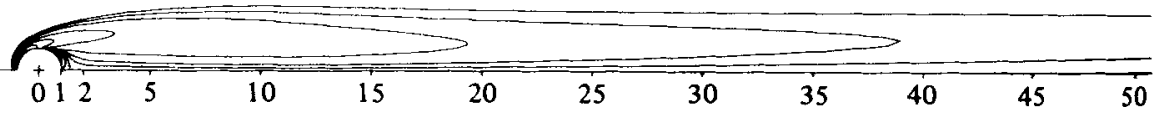

Fugure $11(a-f)$. For legend see page 841 .

these changes had on the computed flow fields. We note a slight movement of the line for equal vorticity. The vorticity in this outer area is almost constant and the pointwise change is much smaller than the movement would seem to suggest.

Figures $14(a)-(d)$ show details of the flow pictures (with data taken from the inner grid) close to the body for Reynolds numbers 20 and 300 and figure 15 the distribution of vorticity on the body surface. The structure and position of the separation point is not completely understood. Theory based on the Helmholtz-Kirchhoff model predicts that, as the Reynolds number goes to infinity (Bordetsky 1923) the separation point may move forward to an angle of $55^{\circ}$ (measured from the front). Our results (and this possible limit) are shown in figure 16 . The width of the wake bubble and the position of its end (measured from the centre of the circle) are shown in figures $17(a, b)$. Table 3 compares the length with earlier results.

There are several ways to calculate the pressure in the flow field and the pressure on the body surface in particular. In $\xi, \eta$ co-ordinates, the pressure satisfies $\left(r=e^{\pi \xi}\right)$

$$
\begin{aligned}
& p_{\xi}=-\frac{2}{R e} \omega_{\eta}+\left\{\Psi_{\xi}^{\prime} \Psi_{\eta \eta}-\Psi_{\eta} \Psi_{\xi \eta}+\pi\left(\Psi_{\xi}^{2}+\Psi_{\eta}^{2}\right)\right\} /(\pi r)^{2}, \\
& p_{\eta}=\frac{2}{R e} \omega_{\xi}+\left\{\Psi_{\eta} \Psi_{\xi \xi}-\Psi_{\xi} \Psi_{\xi \eta}\right\} /(\pi r)^{2},
\end{aligned}
$$



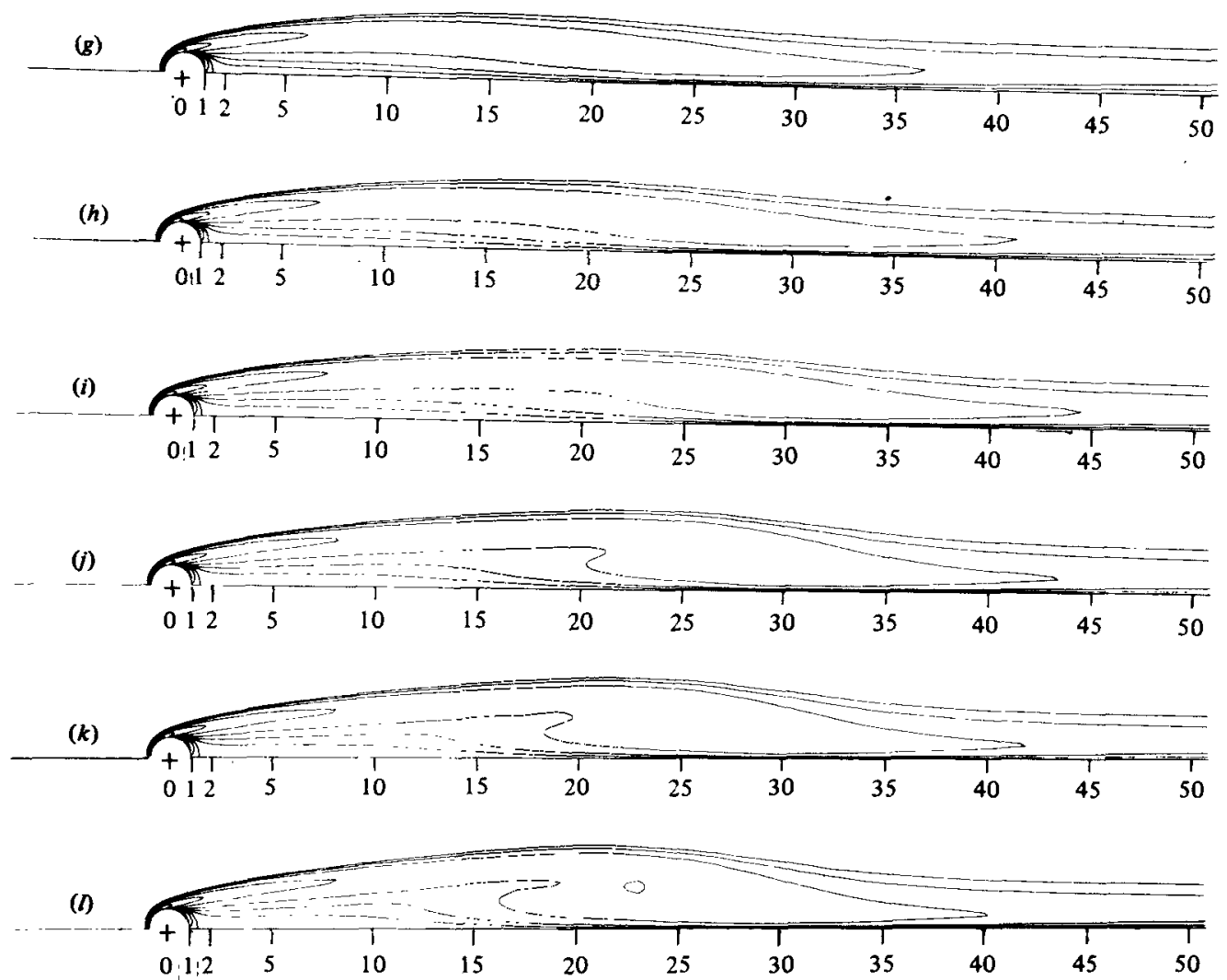

Figure 11. Vorticity fields at different Reynolds numbers. For $(a)-(l)$ see figure 10.

and the Poisson equation

$$
-\left(p_{\xi \xi}+p_{\eta \eta}\right)=\frac{2}{r^{2}}\left\{\left(\frac{\Psi_{\xi \eta}}{\pi}-\Psi_{\eta}\right)^{2}-\left(\frac{\Psi_{\xi \xi}}{\pi}-\Psi_{\xi}\right)\left(\frac{\Psi_{\eta \eta}}{\pi}+\Psi_{\xi}\right)\right\}
$$

with boundary conditions

$$
\left.\begin{array}{rl}
p_{\xi}=-\frac{2}{R e} \omega_{\eta} & \text { on } \quad \xi=0, \\
p_{\eta}=0 \quad \text { on } & \eta=0 \text { and } \eta=1 \\
p=0 & \text { on } \quad \xi=\xi_{\infty} \quad \text { (infinity). }
\end{array}\right\}
$$

In equation (25), $\Psi$ can be replaced by $\psi$ (with no further changes in the equation).

With $\psi$ and therefore $\Psi$ known from our calculations, we employed three different methods to find the pressure at the front stagnation point:

1. We solved the Poisson equation (25) with boundary conditions (26).

2. We evaluated the lineintegral (23) from far upstream, $\xi=\xi_{\infty}$, to $\xi=0$ with $\eta=1$.

3. We evaluated the line integral (23) from $\xi=\xi_{\infty}$ to $\xi=\xi_{r}$ with $\eta=1$, with (24) along a half-circle to $\xi=\xi_{r}, \eta=0$, with (23) to $\xi=0, \eta=0$, and finally with (24) along the surface to $\xi=0, \eta=1$. Figure 18 shows this path.

Table 4 gives the results obtained with these three methods.

Method 1 seems quite accurate for low Reynolds numbers only. A loss of accuracy for 
(a)

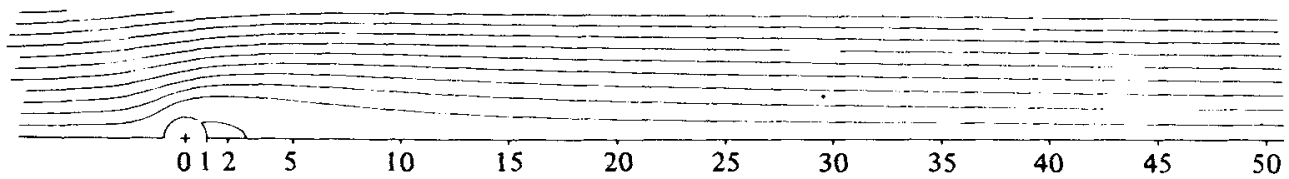

(b)

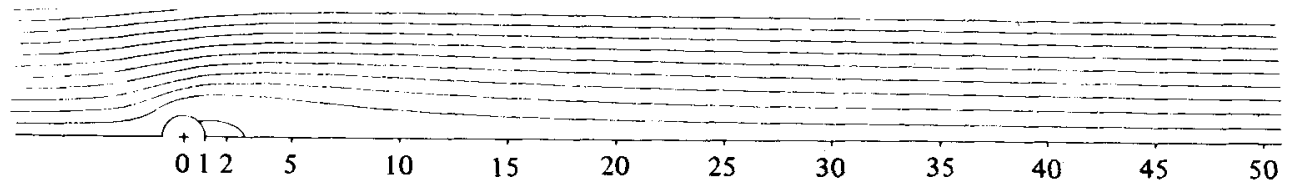

$(c)$

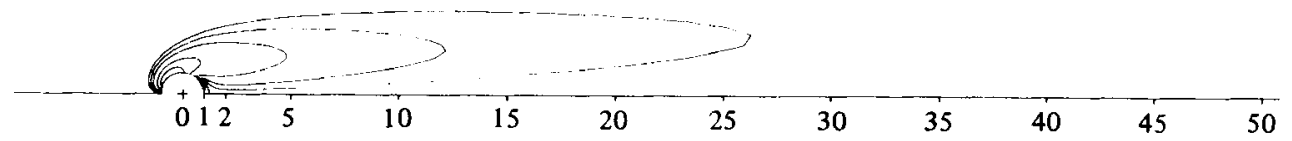

(d)

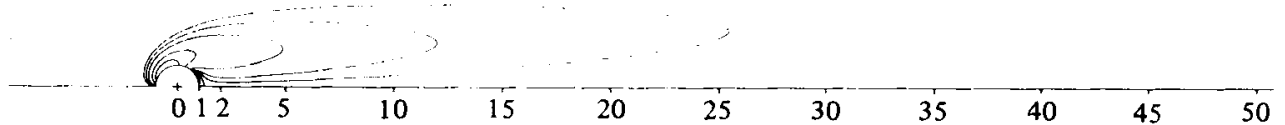

(e)

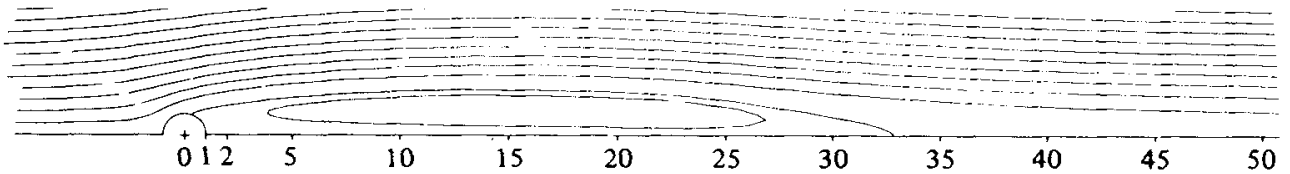

(f)

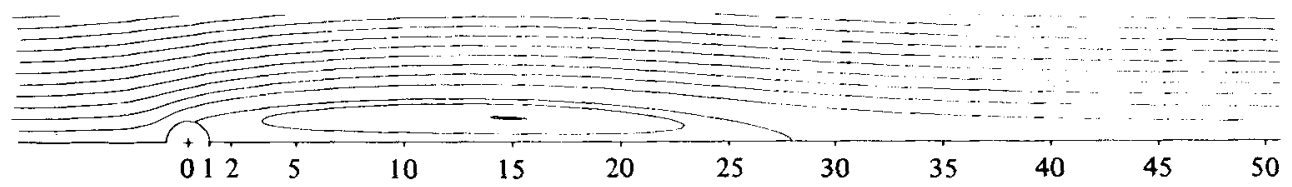

(g)

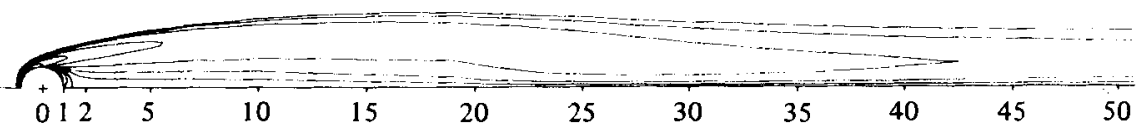

(h)

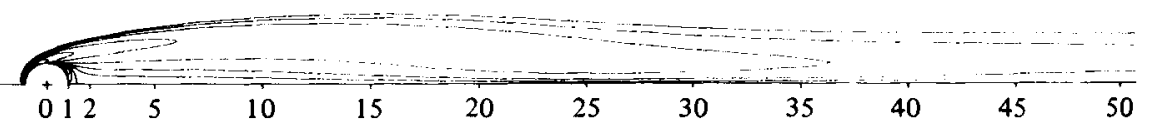

Figure 12. Comparison between results on coarse and fine computational grids. $R e=20,(a)$ coarse grid, $(b)$ fine grid, $(c)$ coarse grid, $(d)$ fine grid. $R e=200$, $(e)$ coarse grid, $(f)$ fine grid, $(g)$ coarse grid, $(h)$ fine grid.

high Reynolds numbers is caused by an increasingly singular behaviour of the righthand side of (25), in particular towards the end of the wake bubble.

Method 2 works well for high Reynolds numbers, but cannot handle low Reynolds numbers well. This is because vorticity is then present quite far in front of the body. This integration can only use values from the outer grid, since the inner grid does not reach the inflow axis. Vorticity was assumed to be absent in this outer region. Although interpolation of $\omega$ from the inner grid was performed, the term $-2 \omega_{\eta} /$ Re was not available with high accuracy.

Method 3 was chosen in order to avoid the problem in the two previous methods. It probably gave the best overall accuracy. A consistency check was obtained by chang- 
(a)

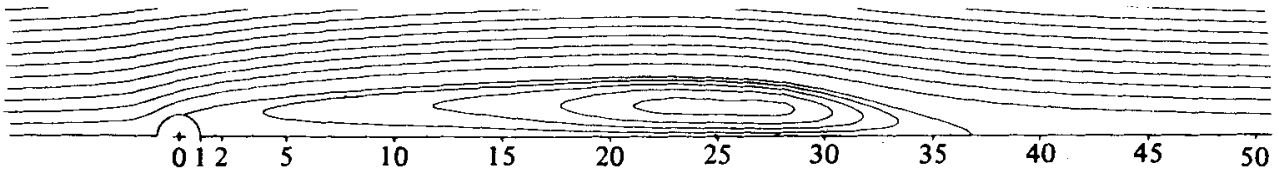

(b)

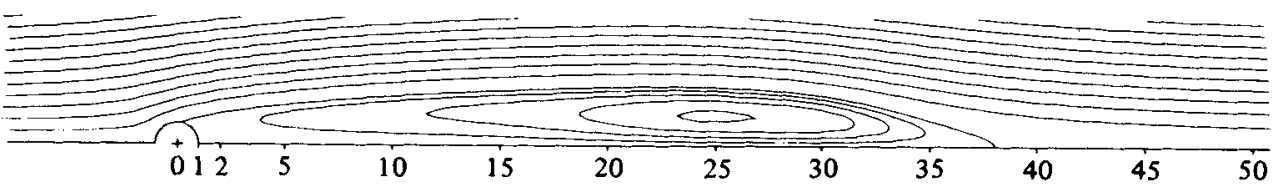

(c)

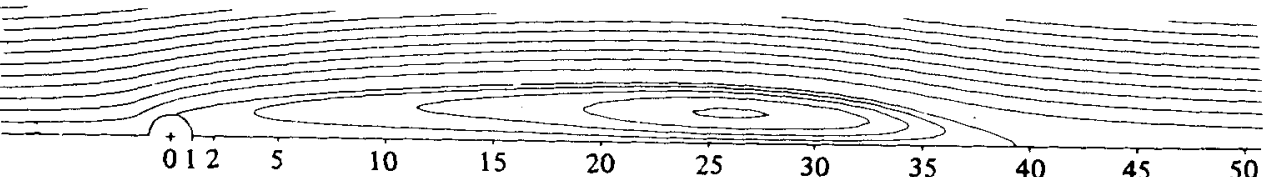

(d)

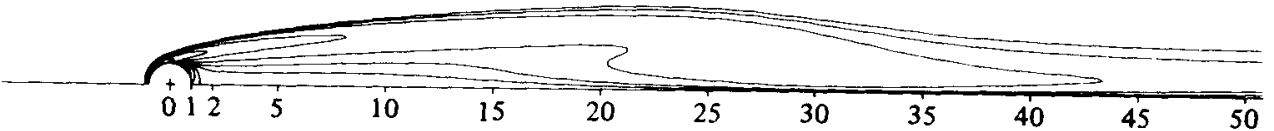

(e)

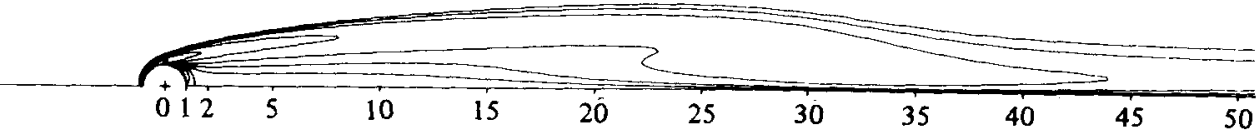

$(f)$

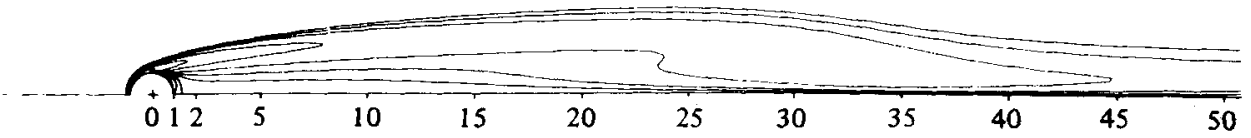

Figure 13. Numerical solution at $R e=290$ for different values of the parameter $c$ in the mapping for the inner grid. $(a) c=0 \cdot 2,\langle b) c=0 \cdot 25,(c) c=0 \cdot 3,(d) c=0 \cdot 2,(e) c=0 \cdot 25,(f) c=0 \cdot 3$.

ing the radius of the outer circular part of the path. In no case did a change within the range $r=2$ to $r=16$ lead to more than a $0.8 \%$ variation in the calculated pressure. The integration from the back to the front stagnation point used data from the inner grid, and should be of high accuracy. For this part of the path equation (24) simplifies to

$$
p_{\eta}=\frac{2}{R e} \omega_{\xi}
$$

since $\Psi_{\xi}$ and $\Psi_{\eta}$ both are zero on the surface.

Table 4 gives the values we have accepted as most likely for the front and back stagnation point pressures. They are also displayed in figure 19. We note that the trend for the rear stagnation pressure appears to be to pass zero towards positive values. This is consistent with Batchelor's limiting solution but is not consistent with the Kirchhoff-Helmholtz solution, where the pressure is zero everywhere inside the wake. (A Reynolds number of 300 is much too low however to give reliable evidence on the final limit.) The pressure distributions over the body surface is shown in figure 20. Table 5 compares the front stagnation pressures we obtained with previously published results. 


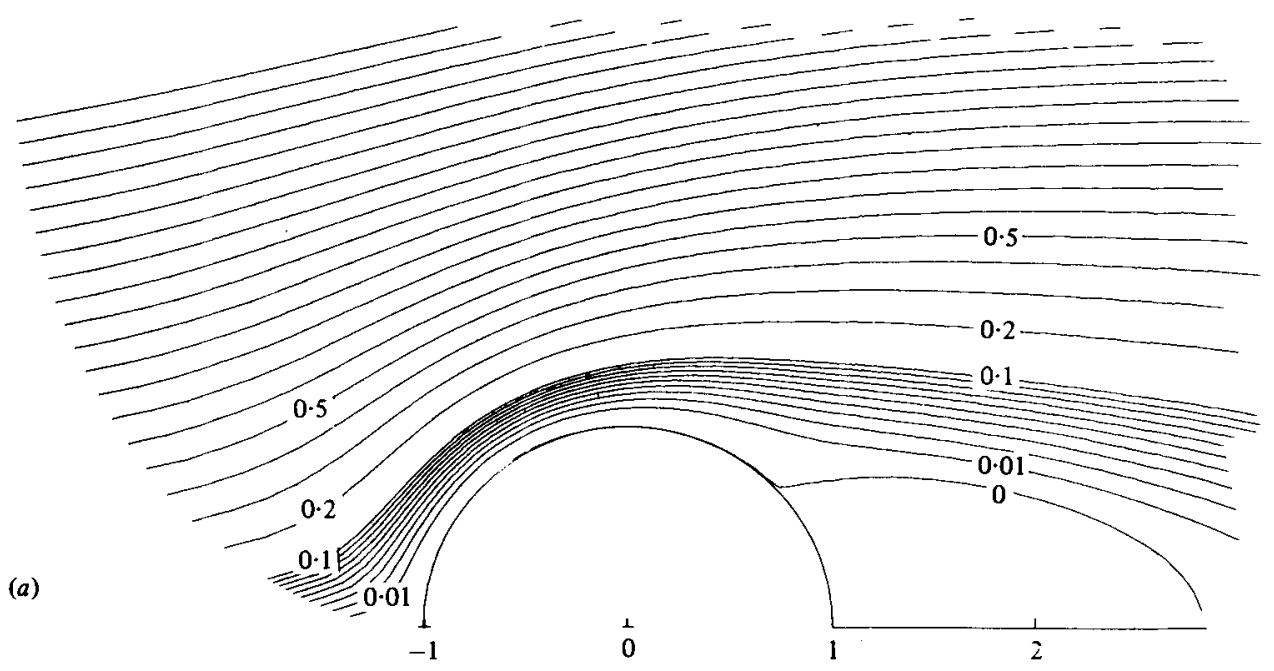

(b)

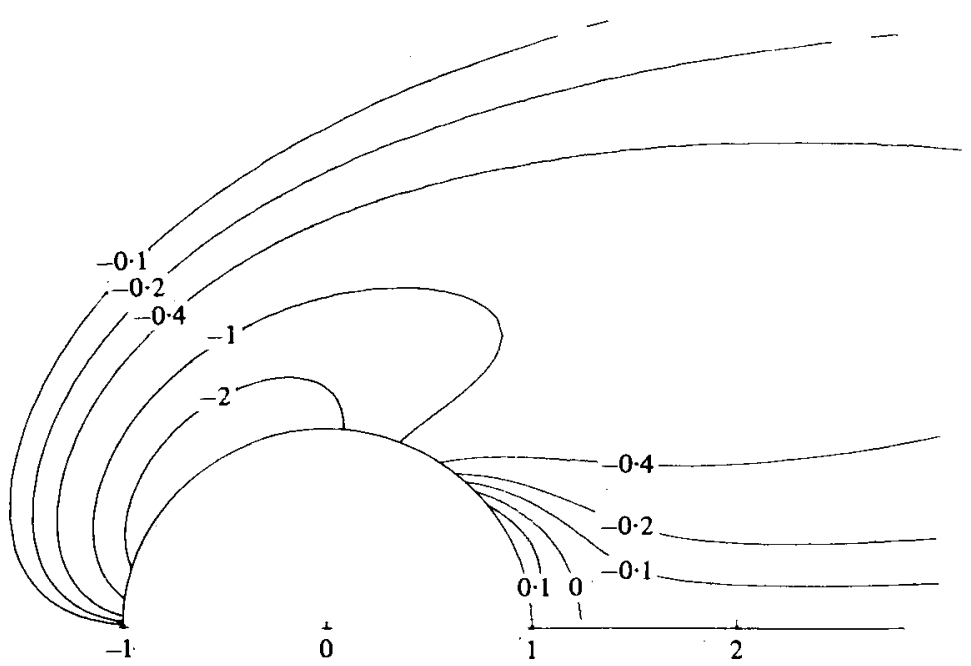

Figure $14(a, b)$. For legend see page 845 .

The drag $D$ on the cylinder is given by

$$
D=\rho U^{2} a C_{D},
$$

where $\rho$ is the fluid density, $U$ the free stream velocity, $a$ the radius of the cylinder and $C_{D}$ the drag coefficient. This non-dimensional coefficient $C_{D}$ can be evaluated by a line integral around the body, choosing for example a circle with radius $r$. The formula for $C_{D}$ is

$$
\begin{aligned}
C_{D}= & \frac{1}{\pi r} \int_{0}^{1}\left\{\left(\Psi_{\xi}^{2}-\Psi_{\eta}^{2}\right) \cos \pi \eta-2 \Psi_{\xi} \Psi_{\eta} \sin \pi \eta\right\} d \eta \\
& -2 r \int_{0}^{1} \Psi_{\eta} \omega \sin \pi d \eta \\
& +4 \frac{r}{R e} \int_{0}^{1}\left(\omega_{\xi}-\pi \omega\right) \sin \pi \eta d \eta .
\end{aligned}
$$




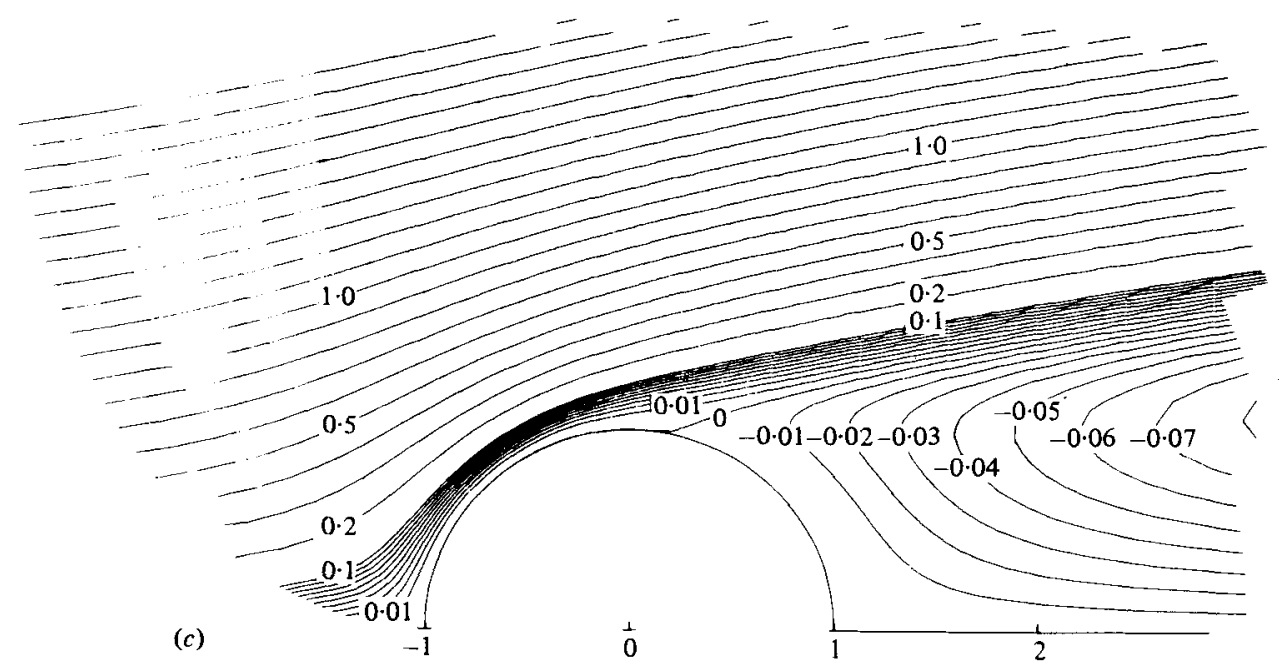

(d)

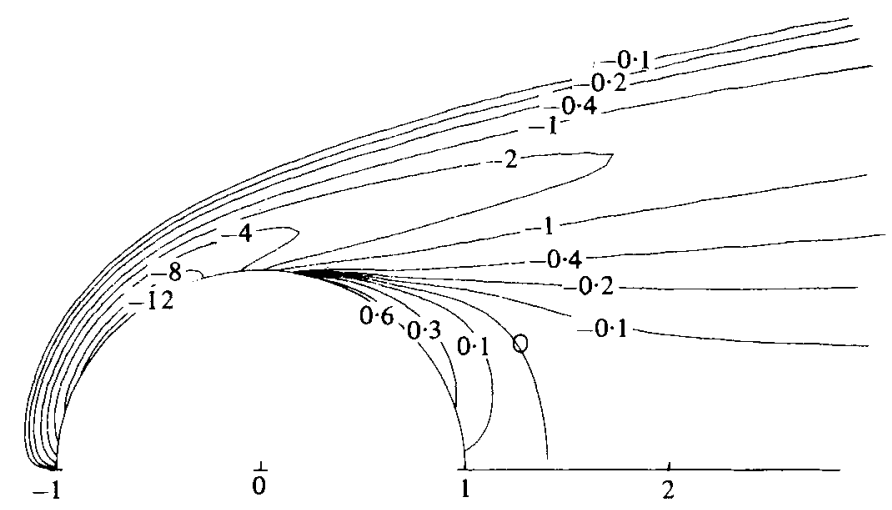

Figure 14. Details of the flow pictures for $R e=20$ and $R e=200 . R e=20,(a)$ stream function, (b) vorticity. $R e=200,(c)$ stream function, $(d)$ vorticity.

Evaluation of $C_{D}$ using different radii $r$ gives to some extent a consistency check on the solution. This integral is however subject to severe numerical cancellations, especially around the end of the wake region at high Reynolds numbers. This can be seen by direct inspection of the integrands. It may also be concluded from the fact that the trapezoidal rule and Simpson's rule on the same data give quite different results (with the first ones more nearly independent of the radius). In figure 21 we show $C_{D}$ as a function of $r$ for $R e 20$ to 300 . With the exception of the last case, there are three curves corresponding to the coarse and fine grids (outer grids $65 \times 52$ and $129 \times 132$ respectively) and the Richardson extrapolated result. The differences between these curves and their independence of $r$ for small radii suggest that

$$
\begin{array}{ll}
\text { at } R e=20, & C_{D}=2 \cdot 0001 \pm 0.0002, \\
\text { at } R e=40, & C_{D}=1 \cdot 4980 \pm 0.0005, \\
\text { at } R e=100, & C_{D}=1 \cdot 058 \pm 0 \cdot 001, \\
\text { at } R e=200, & C_{D}=0.829 \pm 0.002, \\
\text { at } R e=300, & C_{D}=0.722 \pm 0.003 .
\end{array}
$$

These values of $C_{D}$ are displayed in figure 22 . 


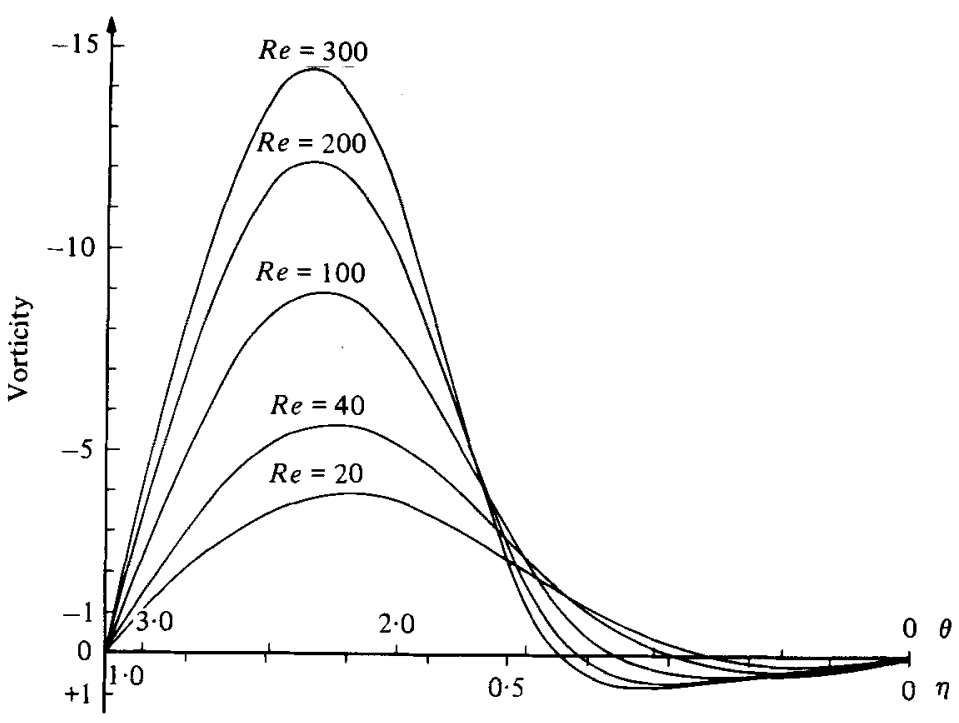

Figure 15. The vorticity distribution over the body surface.

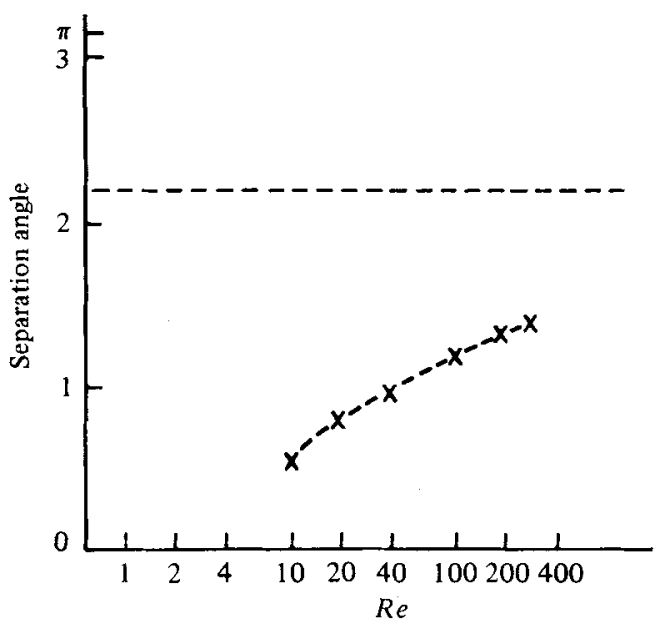

Figure 16. The position of the separation point.

The error estimates are, however, by no means completely certain. For example, they do not take into account possible errors caused by a too restrictive computational region in front of the body for low Reynolds numbers. We do however believe that error to be small.

The irregularity close to the surface in figure 21 comes from the fact that we used values of $\psi$ and $\omega$ from the outer grid only. That grid was designed for evaluation of $\psi$ at large distances and is too coarse for drag calculations close to the surface. Table 6 compares our estimates for $C_{D}$ against others published previously. We believe the discrepancies in almost all cases are mainly due to the inaccurate ways in which the outer boundary conditions have been handled. For example calculations at $R e=2$ 

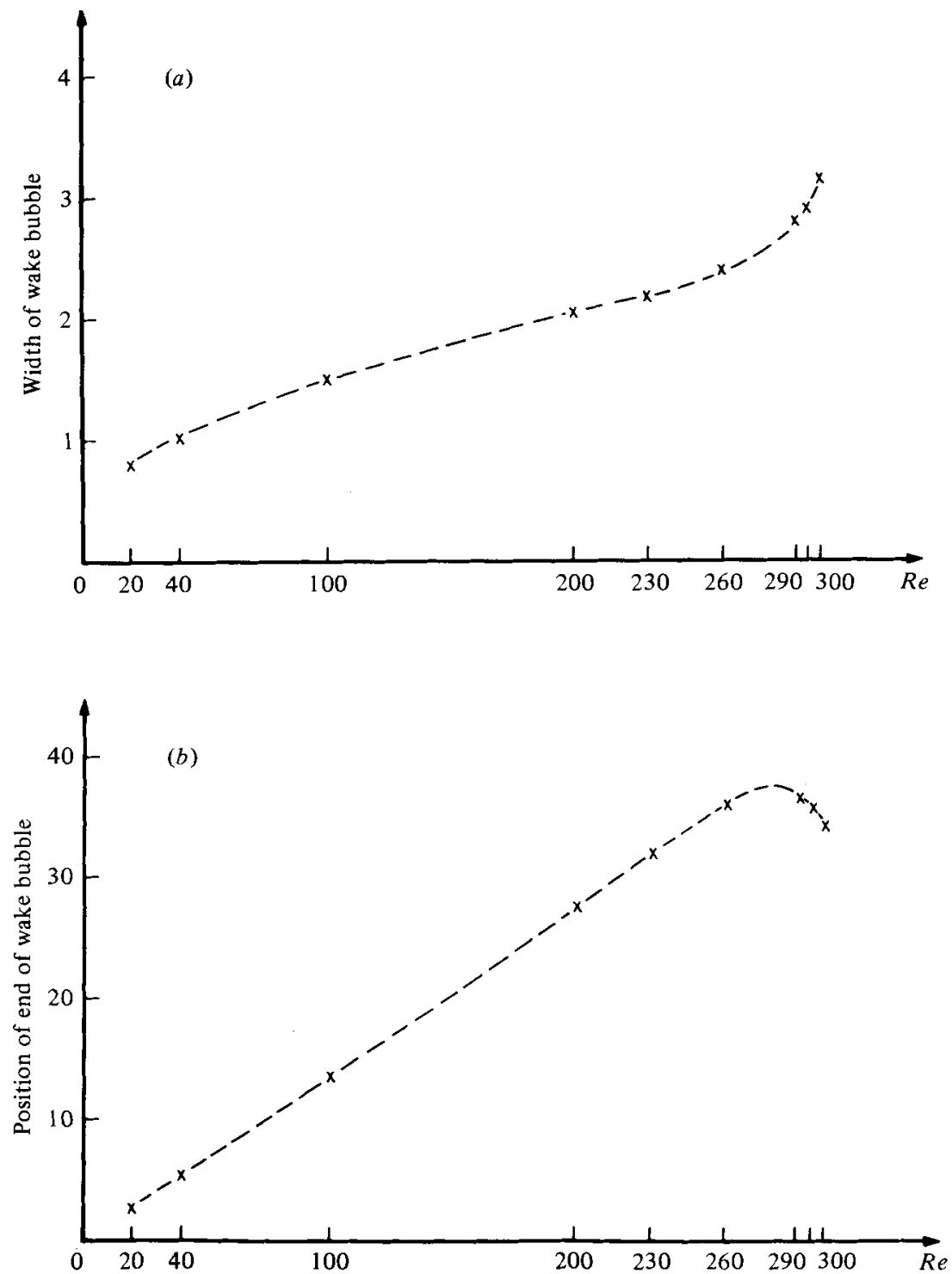

Figure 17. The width $(a)$ and length $(b)$ of the wake bubble. The length is measured from the centre of the cylinder.

showed a change in $C_{D}$ from 7.87 to 6.92 (errors about $19 \%$ and $5 \%$ ) when a freestream boundary condition was applied at $r=23.1$ and $r=91.5$ respectively. The results in closest agreement with ours are those obtained by Dennis (1976) for $R e=20$ and 40 with use of his improved boundary conditions $\left(C_{D}=1.998\right.$ and $C_{D}=1.494$ respectively).

There are several questions pertinent to the flow field which we did not specifically address. For example, there is a possibility that non-unique, steady, perhaps unsymmetric, solutions for high Reynolds numbers may exist. We did not detect any evidence of simple bifurcations or turning points on the main solution branch up to 
吕11蓠|1

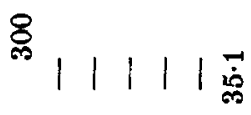

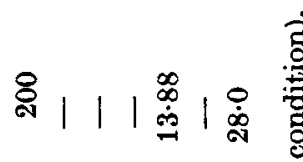

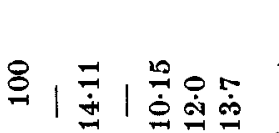

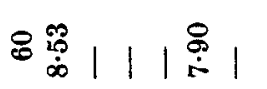

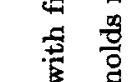

通

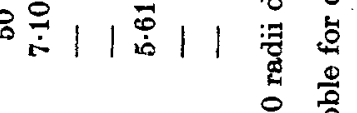

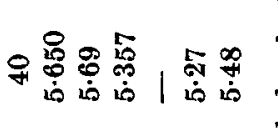

ลิ

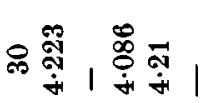

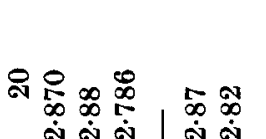

ธक ஸ் ஸे ஸे ஸे

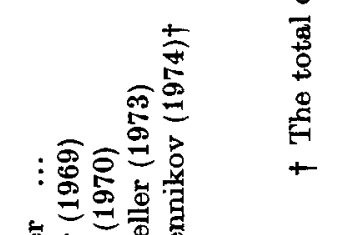

它

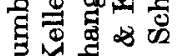

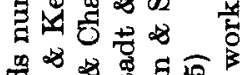

象青

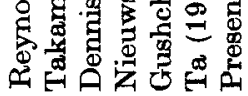




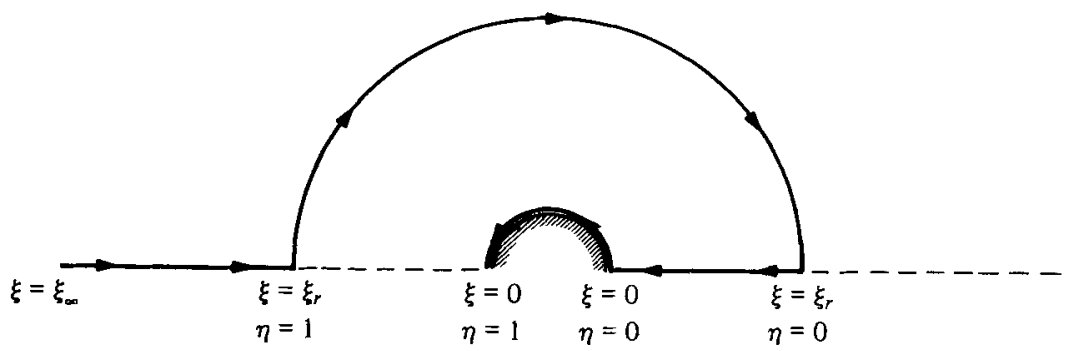

Figure 18. The integration path for the pressure calculation.
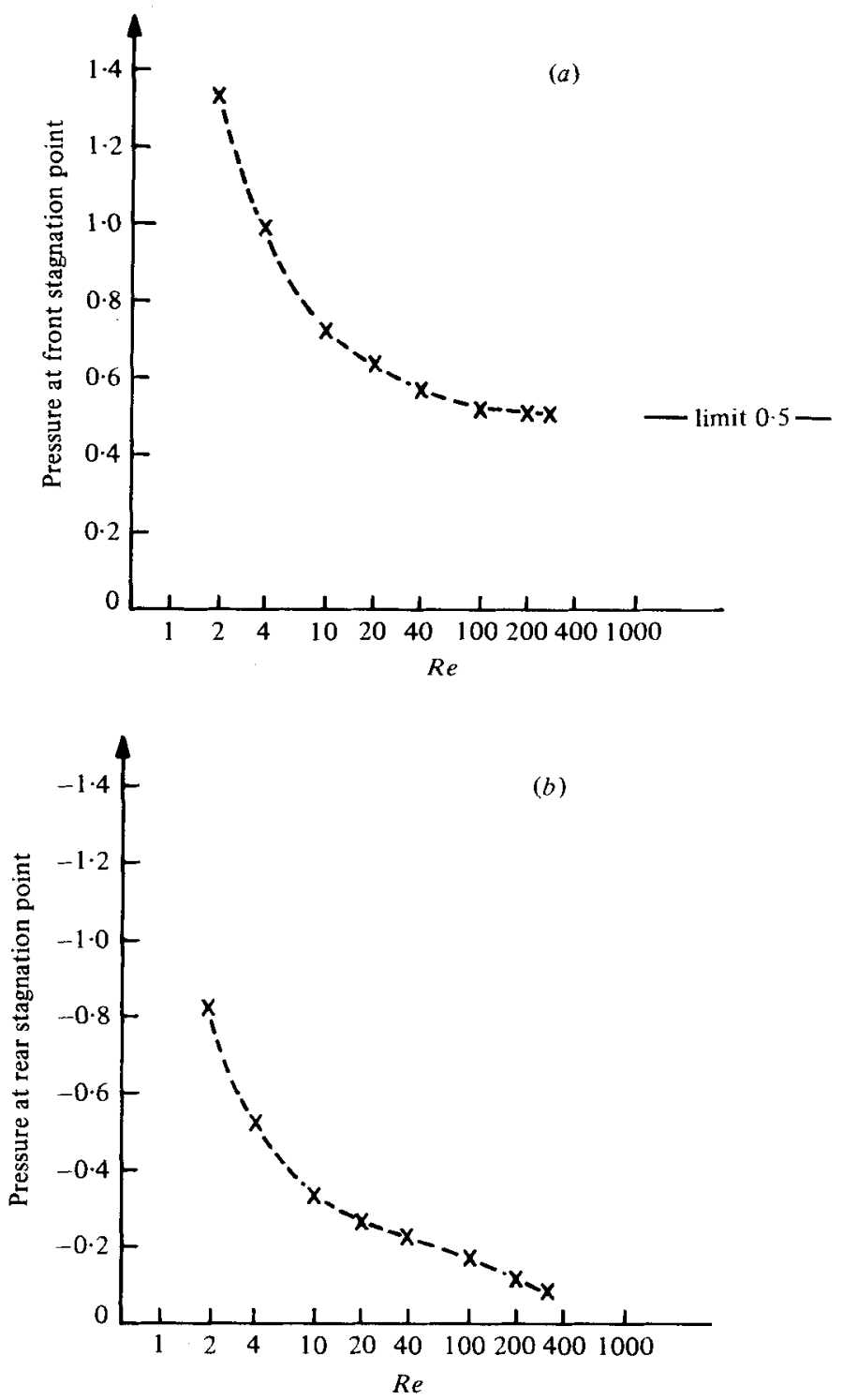

Figure 19. The pressure at $(a)$ the front stagnation point and $(b)$ the rear stagnation point. 


\begin{tabular}{|c|c|c|c|c|c|}
\hline \multirow[b]{3}{*}{$\begin{array}{l}\text { Reynolds } \\
\text { number }\end{array}$} & \multicolumn{4}{|c|}{ Pressure at front stagnation point } & \multirow{3}{*}{$\begin{array}{c}\text { Pressure at } \\
\text { rear } \\
\text { stagnation } \\
\text { point }\end{array}$} \\
\hline & \multicolumn{3}{|c|}{ Method } & \multirow{2}{*}{$\begin{array}{c}\text { Probable } \\
\text { correct } \\
\text { value }\end{array}$} & \\
\hline & 1 & ${ }_{2}$ & 3 & & \\
\hline 20 & $0 \cdot 649$ & $0 \cdot 604$ & $0 \cdot 640$ & $0 \cdot 64$ & -0.27 \\
\hline 40 & 0.586 & 0.562 & 0.571 & 0.57 & -0.23 \\
\hline 100 & 0.543 & 0.528 & 0.523 & 0.53 & -0.17 \\
\hline 200 & 0.490 & $0 \cdot 514$ & 0.506 & 0.51 & $-0 \cdot 12$ \\
\hline 300 & 0.415 & 0.508 & $0 \cdot 499$ & 0.51 & -0.09 \\
\hline
\end{tabular}

TABle 4. Pressure at stagnation points calculated by different methods.

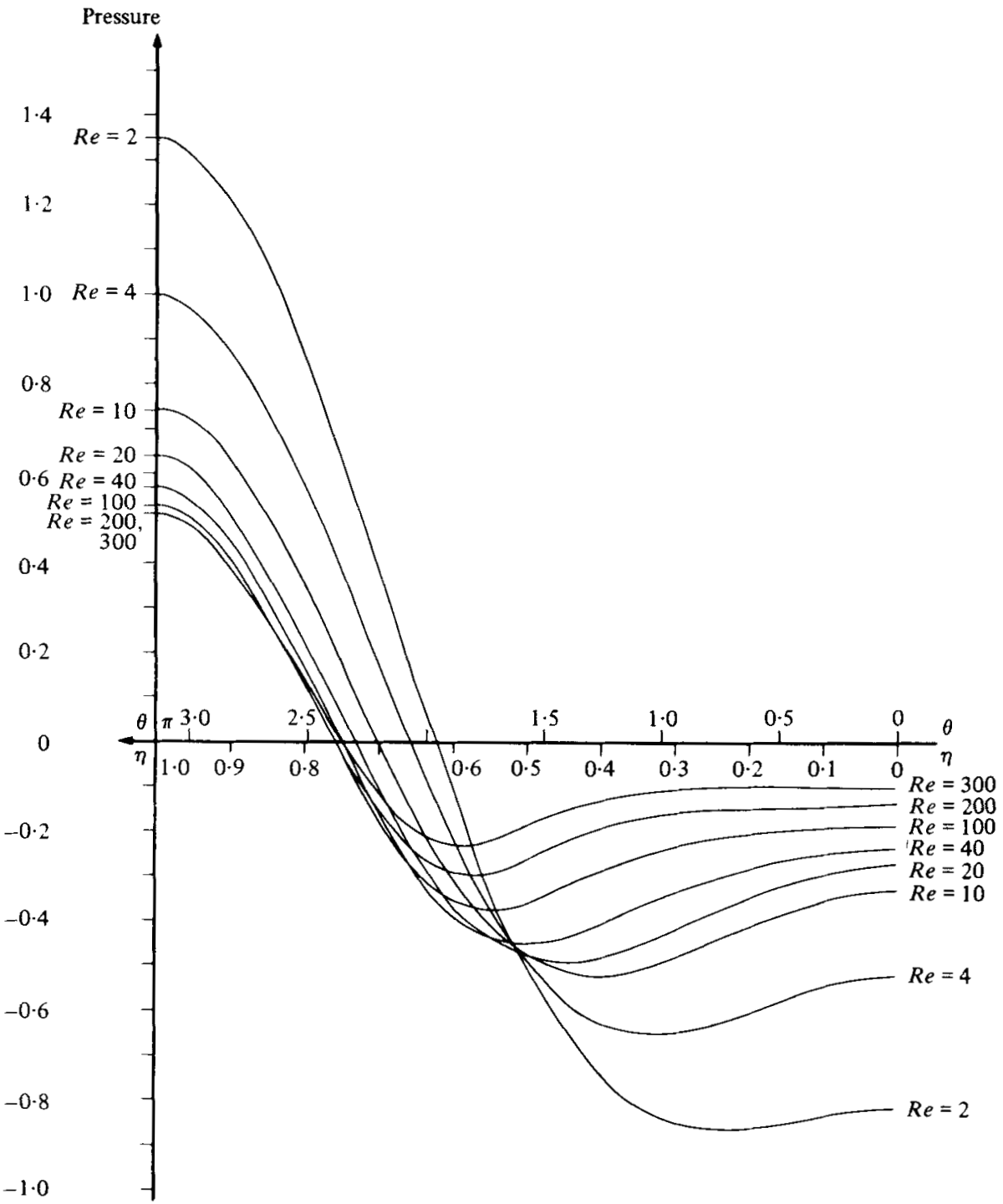

Figure 20. The distribution of pressure over the body surface. 
Steady flow past a circular cylinder

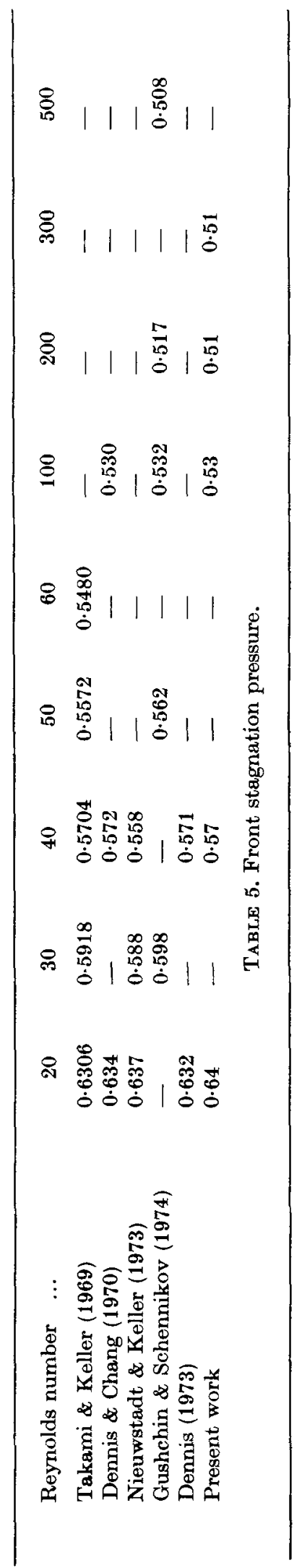




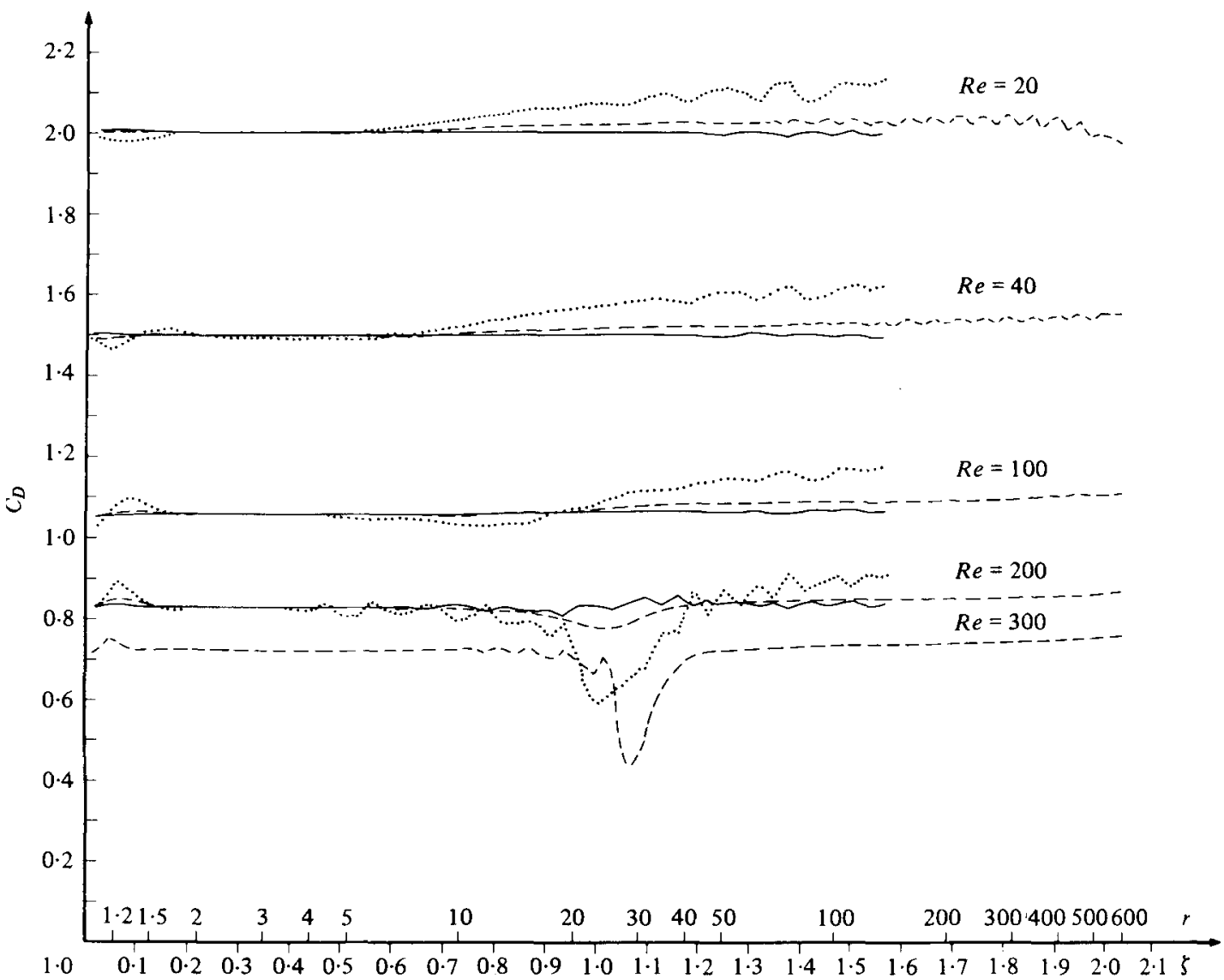

Figure 21. The computed drag coefficients $C_{D}$ as functions of Reynolds number and radius of integration path. ..., coarse grid; ---, fine grid; - - , extrapolation.

$R e=300$. Any such singularities would most likely have led to a breakdown or slowing up of the inner-outer iteration or a singularity of the Jacobian matrix in the Newton method. We monitored the determinant of this matrix and figure 23 shows the logarithm of it. Although we did not find any evidence of non-uniqueness we cannot exclude the possibility of entirely different classes of solutions, even at Reynolds numbers lower than $R e=300$.

This research was supported by Control Data Corporation and by D.O.E. (Office of Basic Energy Sciences). 


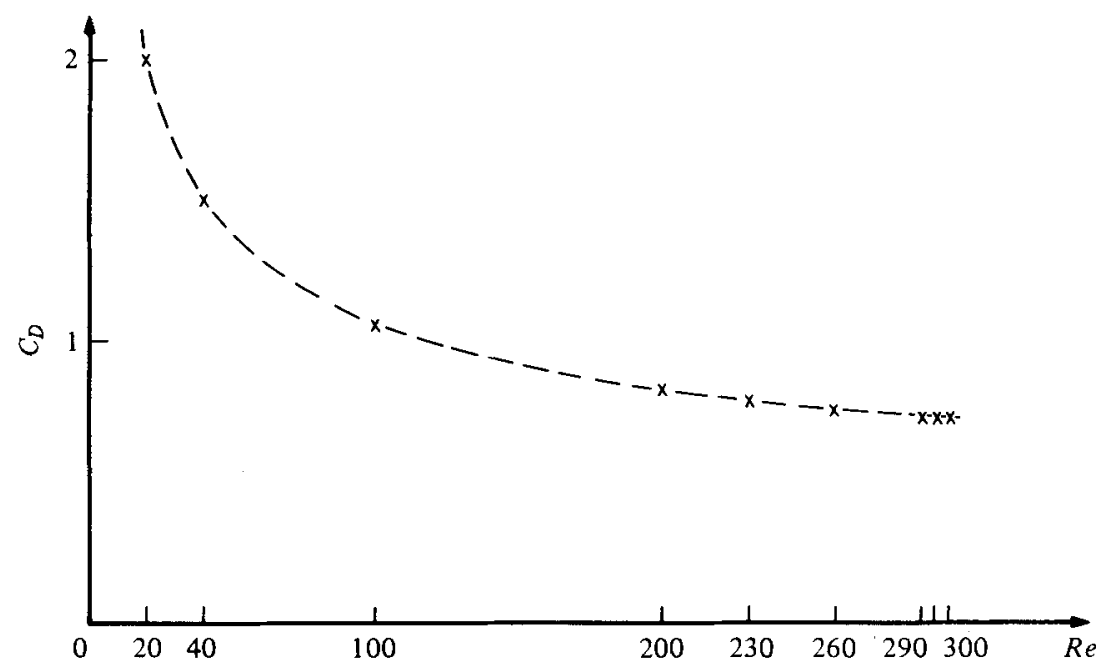

Figure 22. The drag coefficient as a function of Reynolds number.

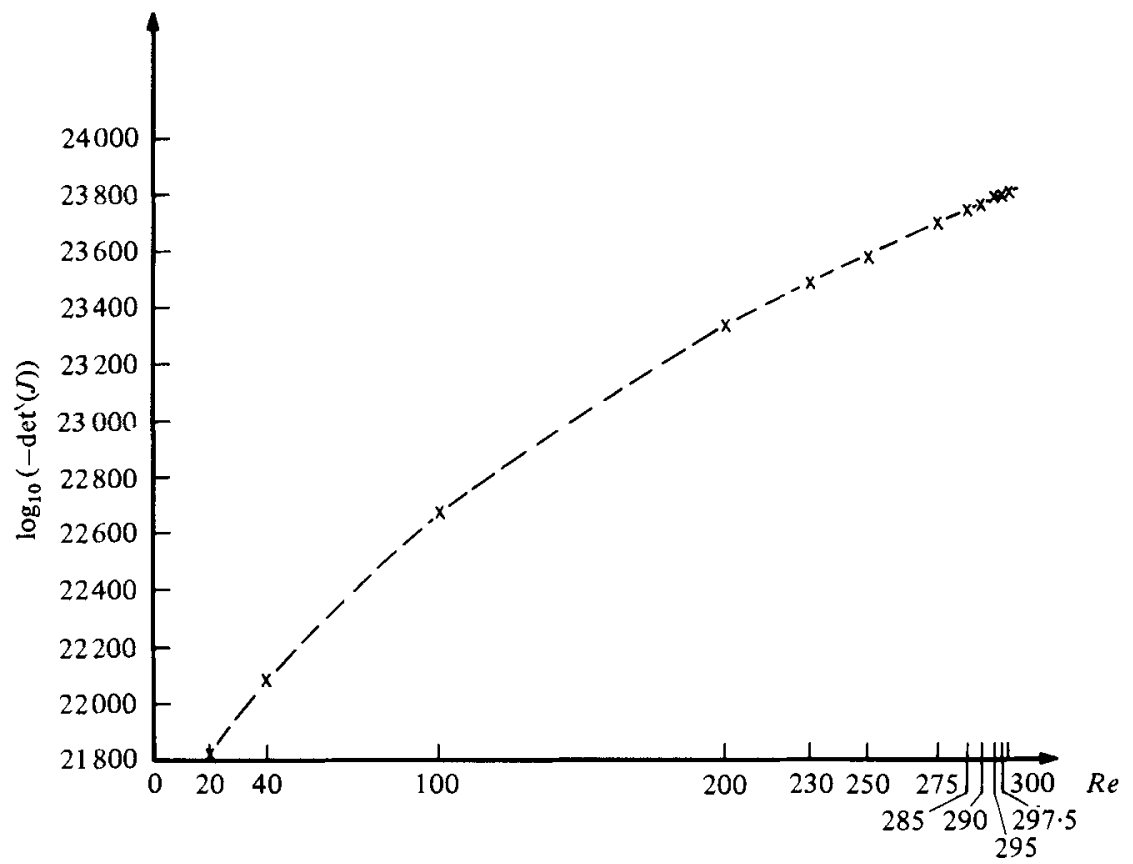

Figure 23. The determinant of the Jacobian matrix for different Reynolds numbers.

\section{REFERENCES}

Allen, D. N. DE G. \& Southwell, R. V. 1955 Relaxation methods applied to determine the motion, in two dimensions, of a viscous fluid past a fixed cylinder. Quart. J. Mech. Appl. Math. 8, 129.

Batchelor, G. K. 1956 A proposal concerning laminar wakes behind bluff bodies at large Reynolds number. J. Fluid Mech. 1, 388.

Brodetsky, S. 1923 Discontinuous fluid motion past circular and elliptic cylinders. Proc. Roy. Soc. A 102, 542 . 
Dennis, S. C. R. 1973 The numerical solution of the vorticity transport equation. Proc. 3rd Int. Conf. on Numerical Methods in Fluid Mech., vol. 2 (ed. H. Cabannes \& R. Tewam), Lecture notes in Physics, vol. 19, p. 120. Springer.

Dennis, S. C. R. 1976 A numerical method for calculating steady flow past a cylinder. Proc. 5th Int. Conf. on Numerical Methods in Fluid Dynamics (ed. A. I. van de Vooren \& P.J. Zandbergen), Lecture notes in Physics, vol. 59, p. 165. Springer.

Dennis, S. C. R. \& Chang, G. Z. 1970 Numerical solutions for steady flow past a circular cylinder at Reynolds numbers up to 100. J. Fluid Mech. 42, 471.

Gushohin, V. A. \& Schennikov, V. V. 1974 A numerical method of solving the Navier-Stokes equations. Zh. vychist. Mat. mat. Fiz. 14, 512.

Hamielec, A. E. \& RAal, J. D. 1969 Numerical studies of viscous flow around circular cylinders. Phys. Fluids 12, 11.

Hazmhoztz, H. v. 1868 Über discontinuirliche Flüssigkoits-Bewegungen, Phil. Mag. 36 (4), 337.

IMAI, I. 1951 On the asymptotic behaviour of viscous fluid flow at a great distance from a cylindrical body, with special reference to Filon's paradox. Proc. Roy. Soc. A $208,487$.

INGHAM, D. B. 1968 Note on the numerical solution for unsteady viscous flow past a circular cylinder. J. Fluid Mech, 31, 815.

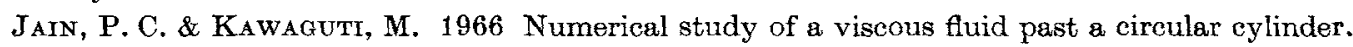
J. Phys, Soc. Japan 21, 2055.

Jain, P. C. \& Sankara Rao, K. 1969 Numerical solution of unsteady viscous incompressible fluid flow past a circular cylinder. Phys. Fluids Suppl. II 57.

Kawagutr, M. 1953 Numerical solution of the Navier-Stokes equations for the flow around a circular cylinder at Reynolds number 40. J. Phys. Soc. Japan 8, 747.

KelleR, H. B. \& TAKamt, H. 1966 Numerical studies of viseous flow about cylinders. In Numerical Solutions of Nonlinear Differential Equations (ed. D. Greenspan), p. 115. Wiley.

Krrсннот, G. 1869 Zur Theorie freier Flüssigkeits-Strahlen. J. reine angew. Math. 70, 289.

LEONARD, B. P. 1979 A stable and accurate convective modelling procedure based on quadratic upstream interpolation. Comp. Meth. Appl. Mech. Engng 19, 59.

Nieuwstadt, F. \& Keller, H. B. 1973 Viscous flow past circular cylinders. Comp. Fluids. 1, 59.

PAtEL, V. A. 1976 Time-dependent solutions of the viscous incompressible flow past a circular cylinder by the method of series truncation. Comp. Fluids 4, 13.

Payne, R. B. 1958 Calculations of unsteady viscous flow past a circular cylinder. J. Fluid Mech. 4, 81 .

RoAche, P.J. 1976 Computational Fluid Dynamics. Albuquerque: Hermosa.

SmTH, F. T. 1979 Laminar flow of an incompressible fluid past a bluff body : the separation, reattachment, eddy properties and drag. J. Fluid Mech. 92, 171.

Son, J. S. \& HanRatTy, T. J. 1969 Numerical solution for the flow around a cylinder at Reynolds numbers of 40, 200 and 500. J. Fluid Mech. 35, 369.

'TA, P. L. 1975 Étude numérique de l'écoulement d'un fluide visqueux incompressible autour d'un cylindre fixe ou en rotation. Effet Magnus. J. Méc. 14, 109.

Takami, H. \& Keller, H. B. 1969 Steady two-dimensional viscous flow of an incompressible fluid past a circular cylinder. Phys. Fluids Suppl. II51.

Thом, A. 1933 The flow past circular cylinders at low speeds. Proc. Roy. Soc. A $141,651$.

Thoman, D. C. \& SzewczyK, A. A. 1969 Time dependent viscous flow over a circular cylinder. Phys. Fluids Suppl. II76.

Tuann, S.-Y. \& Olson, M. D. 1978 Numerical studies of the flow around a circular cylinder by a finite element method. Comp. Fluids 6, 219.

UNDERwood, R. L. 1969 Calculations of incompressible flow past a circular cylinder at moderate Reynolds numbers. J. Fluid Mech. 37, 95 . 